\title{
BMJ Open Impact of obstetric unit closures, travel time and distance to obstetric services on maternal and neonatal outcomes in high-income countries: a systematic review
}

\author{
Reem Saleem Malouf (D) , ${ }^{1}$ Claire Tomlinson, ${ }^{2}$ Jane Henderson, ${ }^{1}$ \\ Charles Opondo (D) , ${ }^{1}$ Peter Brocklehurst, ${ }^{1}$ Fiona Alderdice, ${ }^{1}$ Angaja Phalguni, ${ }^{2}$ \\ Janine Dretzke ${ }^{2}$
}

To cite: Malouf RS, Tomlinson C, Henderson J, et al. Impact of obstetric unit closures, travel time and distance to obstetric services on maternal and neonatal outcomes in high-income countries: a systematic review. BMJ Open 2020;10:e036852. doi:10.1136/ bmjopen-2020-036852

- Prepublication history and additional material for this paper is available online. To view these files, please visit the journal online (http://dx.doi.org/10. 1136/bmjopen-2020-036852)

Received 13 January 2020 Revised 07 September 2020 Accepted 23 September 2020

Check for updates

(C) Author(s) (or their employer(s)) 2020. Re-use permitted under CC BY. Published by BMJ.

${ }^{1}$ Nuffield Department of Population Health, Policy Research Unit in Maternal Health and Care, National Perinatal Epidemiology Unit, University of Oxford, Oxford, UK

${ }^{2}$ Institute of Applied Health Research, College of Medical and Dental Sciences, University of Birmingham, Birmingham, UK

Correspondence to Dr Reem Saleem Malouf; reem.malouf@npeu.ox.ac.uk

\section{ABSTRACT}

Objectives To systematically review (1) The effect of obstetric unit (OU) closures on maternal and neonatal outcomes and (2) The association between travel distance/ time to an $\mathrm{OU}$ and maternal and neonatal outcomes.

Design Systematic review of any quantitative studies with a comparison group.

Data sources Embase, MEDLINE, PsycINF0, Applied Social Science Index and Abstracts, Cumulative Index to Nursing and Allied Health and grey literature were searched.

Methods Eligible studies explored the impact of closure of an $\mathrm{OU}$ or the effect of travel distance/time on prespecified maternal or neonatal outcomes. Only studies of women giving birth in high-income countries with universal health coverage of maternity services comparable to the UK were included. Identification of studies, extraction of data and risk of bias assessment were undertaken by at least two reviewers independently. The risk of bias checklist was based on the Cochrane Effective Practice and Organisation of Care criteria and the Newcastle-Ottawa scale. Heterogeneity across studies precluded meta-analysis and synthesis was narrative, with key findings tabulated.

Results 31 studies met the inclusion criteria. There was some evidence to suggest an increase in babies born before arrival following OU closures and/or associated with longer travel distances or time. This may be associated with an increased risk of perinatal or neonatal mortality, but this finding was not consistent across studies. Evidence on other maternal and neonatal outcomes was limited but did not suggest worse outcomes after closures or with longer travel times/ distances. Interpretation of findings for some studies was hampered by concerns around how accurately exposures were measured, and/or a lack of adjustment for confounders or temporal changes.

Conclusion it is not possible to conclude from this review whether OU closure, increased travel distances or times are associated with worse outcomes for the mother or the baby.

PROSPERO registration number CRD42017078503.
Strengths and limitations of this study

- This review is the first to synthesise systematically the current evidence on the impact of closure of obstetric units and of travel distance and travel time to obstetric units on neonatal and maternal outcomes.

- Rigorous systematic review methodology was applied including a sensitive search strategy to ensure all relevant evidence was identified.

- Heterogeneity across included studies precluded any form of meta-analysis.

- A paucity of evidence on a number of outcomes, and methodological concerns for some studies limited conclusions that could be drawn.

\section{BACKGROUND}

Closure of small obstetric units (OUs) and centralisation of obstetric services in larger units has been proposed to increase levels of consultant obstetrician cover to improve safety and limit costs. However, closure of OUs or conversion of OUs to midwifery-led units/community-based services potentially leads to an increase in travel distance or time for women in labour from their home to the nearest OU. Increases in travel time could potentially increase the risk of adverse birth outcomes.

Travel time and distance are widely used as measures to explore the geographical accessibility of health services. ${ }^{1}$ In a systematic review, ${ }^{2}$ the association between travelling further to healthcare facilities and having worse health outcomes was established, but the review did not include studies of maternity care. The impact of OU closure and increase in travel time/distance to the OU on perinatal and maternal outcomes have not been systematically assessed. One 
review $^{3}$ evaluating the effects of regionalisation of perinatal services has been published. This concluded that regionalisation programmes appeared to be correlated with improvements in perinatal outcomes but that the evidence was weak. A narrative review ${ }^{4}$ included 10 studies that explored travel time and distance to and between maternity services and adverse birth outcomes to inform the consultation on maternity services in Wales. The review was limited to studies reported in English and there was no clear association between travel distance or time and adverse birth outcomes

Therefore, uncertainty remains about the association between OU closure, prolonged time or distance to OUs and adverse perinatal outcomes. Specifically, there is a rise in the risk of babies born before arrival (BBA, also referred to as unplanned out of hospital births). Being BBA is more common before term and has been reported to be associated with higher perinatal mortality (PM). ${ }^{5}$ Conversely, Lasswellet et at found neonatal mortality (NM) was reduced when services were configured to ensure very preterm infants are born in a large maternity hospital with neonatal intensive care unit (level III NICU) - In addition to mortality, Apgar scores (a standardised measure of the physical condition of a newborn infant) and neonatal admission to intensive care provide an indication of perinatal infant health.

The impact on maternal outcomes is also unclear. There are concerns that low-risk women who give birth in larger hospitals may experience more interventions, for example, increased frequency of caesarean section (CS). ${ }^{7}$ Along with CS, evidence on maternal mortality (MM) and maternal birth complications such as postpartum haemorrhage (PPH) and maternal blood transfusion, was also sought in this review to identify the potential impact of OU closure on maternal outcomes.

In this review, we aimed to systematically identify, critically appraise and synthesise the evidence relating to: (1) The effect of OU closures on maternal and neonatal outcomes (compared with the surrounding area or a comparable population) and (2) The association between travel distance or time to an $\mathrm{OU}$ and maternal and neonatal outcomes.

\section{REVIEW METHOD}

The Meta-Analyses and Systematic Reviews of Observational Studies in Epidemiology (MOOSE) reporting guideline was followed. ${ }^{8}$

\section{Criteria for considering studies for this review \\ Types of studies}

Any quantitative study design with a comparison group was eligible for inclusion. Studies were included from 1990 onwards. The year 1990 was chosen as a cut-off date because significant advances were made in neonatal care in the early 1990s, such as surfactant therapy, assisted ventilation, prophylactic infection control and antenatal steroid therapy, which impacted on the delivery of maternity services. ${ }^{9}$ The quantitative components of mixed methods studies were also eligible. Studies were included if they:

- Explored the impact of closure of an OU on maternal or neonatal outcomes either in a before-and-after comparison (same population catchment area), or a geographical comparison of different areas (comparable populations).

And/or

- Compared maternal and neonatal outcomes after an OU closure and retention or creation of midwifery led units to replace the OU.

- Explored the effect of travel time and/or distance on maternal and neonatal outcomes providing at least two travel times and/or distances from women's homes to the nearest OU.

- Explored maternal and neonatal outcomes following maternal transfer from planned or unplanned home birth to the nearest maternity centre.

We included studies of women giving birth in highincome, the Organisation for Economic Co-operation and development (OECD) countries with universal health coverage (UHC) of maternity services comparable to the UK. The list of OECD countries is shown in online supplemental appendix 1. UHC is defined as healthcare that meets everyone's right to access high quality essential health services where and when they need them without financial difficulty. ${ }^{10}$

\section{Types of exposures}

OU closure: the closure of an OU was compared with no closure of an OU for the same or comparable geographical catchment areas prior to the closure. For a study comparing different geographical areas affected by the closure of an OU, the least affected area was used as a control group. For the purpose of this review, we used the definition of an OU used in the Birthplace Research programme in England, ${ }^{11}$ which defined an OU as 'a clinical location in which care is provided by a team, with obstetricians taking primary professional responsibility for women at high risk of complications during labour and birth. Midwives offer care to all women in an OU, whether or not they are considered at high or low risk, and take primary responsibility for women with straightforward pregnancies during labour and birth. Diagnostic and treatment medical services including obstetric, neonatal and anaesthetic care are available on site, 24 hours a day'11 (P12).

Travel distance or time to the nearest OU: a shorter travel distance or time was compared with a longer travel distance or time. We used the definition of a shorter or a longer time or distance as defined by the included studies. When a study compared several different travel times or distances to the nearest OU, those with the shortest travel distance or time were used as the control group.

The following types of studies were excluded:

- Studies comparing maternal and or neonatal outcomes based on hospital size, level of NICU, type 
of hospital or model of care (eg, caseload midwifery care vs consultant care).

- Studies on regionalisation of neonatal care (number of centres with NICUs).

- Studies where a proximity rather than the actual travel time or travel distance was given (eg, rural vs urban, remote vs very remote areas).

- Studies which did not report at least one of the outcomes.

\section{Review outcomes}

The following outcomes were predefined in the study protocol:

\section{Maternal outcomes}

Maternal mortality (MM), caesarean section (CS) (overall, emergency or intrapartum), severe perineal trauma (including third and fourth degree tears), postpartum haemorrhage (PPH), maternal admission to intensive care units (ICU) and maternal blood transfusion.

\section{Neonatal outcomes}

Stillbirth (SB) (overall or intrapartum), neonatal mortality (NM), PM, infant mortality (IM), babies BBA, neonatal unit admission (NNU), Apgar score and hypoxic-ischaemic encephalopathy (HIE).

\section{REVIEW METHODS}

A comprehensive search strategy was developed in collaboration with an information specialist (NR). We searched Embase, Medline, PsycINFO, Applied Social Science Index and Abstracts and Cumulative Index to Nursing and Allied Health databases (from 1990 to February 2019). We also searched the grey literature in the databanks of British Library EThOS, Open Grey and ProQuest Dissertations \& Theses Global. National Health Service (NHS) Trusts and Health Boards in the UK were also contacted where we had been able to identify an OU closure to request information about any evaluations that were conducted. The references of eligible studies and relevant reviews were checked to identify additional studies not retrieved by the search. Searches were based on index terms and text words relating to the population/setting (eg, maternity service, pregnancy, neonatal) and exposures (eg, travel/distance or closure/regionalisation). Due to the variable nature of terms and indexing used, the strategy was kept broad by using a range of alternate terms and not limiting by outcome. No language restriction was applied. A sample search strategy for MEDLINE is shown in online supplemental appendix 2.

At least two reviewers (RSM, CT, AP, FA and JH) independently screened the references for relevance against the review eligibility criteria using Eppi-reviewer software (V.4) ${ }^{12}$ Full-text study screening was also performed by at least two reviewers (RSM, CT, CO, JH and FA). Disagreements regarding study eligibility were resolved through discussion and consensus within the review team. We contacted authors of relevant studies published as abstracts for further information. Data extraction and risk of bias assessment were undertaken by at least two reviewers (RSM, CT, CO, JH, FA and JD). The risk of bias checklist was adapted from the Effective Practice and Organisation of Care (EPOC) ${ }^{13}$ and the Newcastle-Ottawa scale (for case-control studies). ${ }^{14}$ Risk of bias assessment included selection of study groups, measurement of exposure and outcomes, missing data and appropriateness of analysis (eg, logistic regression analysis). For case-control studies, selection and comparability of cases and controls were also considered. The review team rated the quality of evidence for each domain in the tool as low, high or unclear risk of bias, or yes, no and unclear in meeting quality criteria.

Results were synthesised narratively and the key findings tabulated. The included studies varied in their study design, categories of exposure, outcomes reported, whether adjusted or unadjusted results were presented and factors adjusted for. This clinical and methodological heterogeneity across the included studies precluded any form of meta-analysis. Prespecified subgroups were risk status of woman (low vs high), parity, gestational age, UK studies compared with non-UK studies and planned versus unplanned CS; formal subgroup analyses were, however, not possible. Evidence regarding OU closure, travel distance and travel time is reported separately, and by outcome. We have highlighted where crude (unadjusted) ORs (cOR) and adjusted ORs (adjOR) have been reported.

\section{PATIENT AND PUBLIC INVOLVEMENT}

We involved our parent, patient and public involvement (PPPI) Stakeholders Network, to explore which outcomes were important from a maternal perspective. The dissemination of findings to stakeholders will be through plain language summaries developed with members of our PPPI stakeholder network.

\section{Search results}

Searches of bibliographic databases and other sources from 1990 to February 2019 yielded 13271 unique references and the steps of study selection are presented in the Preferred Reporting Items for Systematic Reviews and Meta-Analyses flow chart (figure 1). The eligibility of 295 full-text articles were assessed independently. Two hundred and sixty articles were excluded for various reasons, including: studies conducted in low-income/ middle-income countries, comparing different models or levels of maternity care, assessing women's transfer from primary to secondary maternity centres, or not providing quantifiable measures of travel time/distance (full list available from authors). Thirty-one studies, reported in 35 articles, met the review eligibility criteria (figure 1 ). One study ${ }^{5}$ included information on both OU closure and travel distance. Ten studies provided information on OU closures, 7 studies compared different travel distances 
PRISMA Flow Diagram

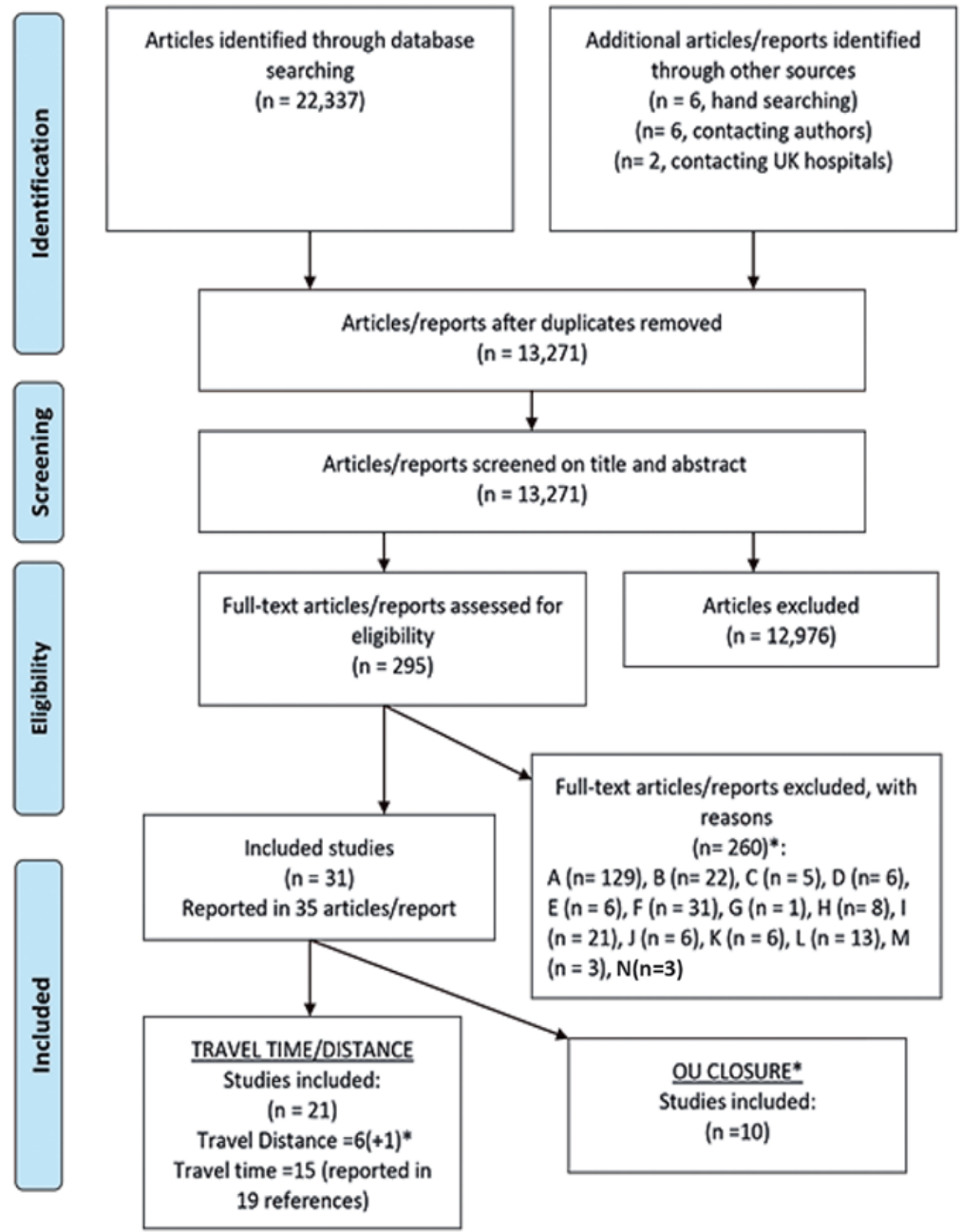

*One study, Blondel 2011 contribute data to both travel distance and closure review questions

From: Moher D, Liberati A, Tetzlaff J, Altman DG, The PRISMA Group (2009). Preferred Reporting Nems for Systematic Reviews and MetaAnalyses: The PRISMA Statement. PLOS Med 6(7): e1000097. doi:10.13711/journal.pmed1000097

For more information, visit www. prisma-statement.org.

Reason for exclusion:

A. Different study designs

B. Studies comparing different models of maternity care

C. Studies comparing different hospital volumes

D. Studies comparing different levels of care

E. Studies comparing different hospital types (private vs. public, local vs. central)

F. Transfer from primary to secondary birth centres

G. Cost of care

H. Neonatal transfer

I. Regionalisation

J. Not in high-income countries or in the USA

K. Distance or travel time proximity

L. No outcomes

M. Modelling studies

N. Not found

Figure 1 PRISMA flow diagram. PRISMA, Preferred Reporting Items for Systematic Reviews and Meta-Analyses.

from women's homes to the nearest OU and 15 studies compared different travel times from women's homes to the nearest OU.
REVIEW RESULTS

Evidence from OU closures

A detailed description of the ten included OU closure 
studies is presented in table 1 . Three studies were from the UK, with two reported as abstracts only ${ }^{1516}$ and one an unpublished data series from East Lancashire Hospitals NHS Trust, UK (East Lancashire Hospitals NHS Trust, unpublished data 2017). There appeared to be overlap between populations reported in two studies (East Lancashire Hospitals NHS Trust, unpublished data 2017) and Fleming et al. ${ }^{15}$ Three studies were from Scandinavia, ${ }^{71718}$ three from Canada ${ }^{19-21}$ and one from France. $^{5}$

Seven studies compared adverse birth outcomes before and after centralisation of services, which included closure of varying numbers of OUs. All three studies from the $\mathrm{UK}^{15} 16$ and (East Lancashire Hospitals NHS Trust, unpublished data 2017), examined the impact of the amalgamation of two OUs. Four studies were published after $2014^{15-1720}$; the earliest was from $1990 .^{21}$ Three studies included all births ${ }^{171821}$; the other studies varied in their eligibility criteria, for example, restricting the analysis to singletons pregnancies, live births, various gestational ages and birth weight, hospital births or location. Reporting of eligibility criteria and participant characteristics across studies was inconsistent (table 1).

\section{Risk of bias assessment}

Risks of bias related to a lack of reporting of whether changes over time (other than closure/reconfiguration) could have influenced the findings, with only two ${ }^{1720}$ of 10 studies reporting that temporal variation was adjusted for in the analysis (table 2). Further, 5 out of 10 studies either did not adjust results for potential confounding factors or provided insufficient information to know whether this was undertaken. Five out of 10 studies did not provide sufficient information to gauge the completeness of data. Half of the studies reported and used appropriate data analysis methods. Other potential sources of bias (eg, relating to selection, exposure and outcome) were less of a concern due to the use of routinely collected registry data before and after the closure and the objective nature of most outcomes.

\section{Findings}

A summary of maternal and neonatal outcomes is presented in table 3 .

\section{Maternal outcomes}

Maternal mortality (MM)

In the two studies that reported MM, ${ }^{16}{ }^{20}$ the number of deaths $(<5)$ was too low to allow comparisons between the preclosure and postclosure groups.

\section{Caesarean section (CS) (overall or intrapartum)}

Total CS rates were reported in four studies. ${ }^{75} 2021$ One UK study ${ }^{15}$ reported a decline in CS rates following the amalgamation of two units from $26.1 \%$ to $21.5 \%$.

A Norwegian study, ${ }^{7}$ reported an increase in CS rates from $13.1 \%$ to $16.4 \%$ following OU closure, (cOR 1.31, $95 \%$ CI 1.27 to 1.35 ) as did two Canadian studies ${ }^{21}$ (cOR
$1.13,95 \%$ CI 1.09 to 1.18 ) and (cOR $1.10,95 \%$ CI 1.01 to 1.19). ${ }^{20}$ No adjusted results were reported.

\section{Emergency CS}

Emergency CS rates were reported in one UK study, ${ }^{16}$ which found no difference before/after the amalgamation of two OUs (cOR $0.95,95 \%$ CI 0.86 to 1.05 ).

\section{Severe perineal trauma (third or fourth degree tear)}

Two studies ${ }^{16} 20$ reported this outcome and found no statistically significant difference between the before/ after closure groups. The incidence of the outcome in both studies was low $(<3 \%)$.

$\mathrm{PPH}-\mathrm{No}$ studies reported this outcome.

\section{Maternal admission to ICU}

Two studies ${ }^{1620}$ found no significant difference before/ after the amalgamation of two OUs in the number of women requiring admission to ICU (cOR 0.80, 95\% CI 0.44 to 1.46$).{ }^{16}$ The numbers in one study ${ }^{20}$ were too small $(<5)$ to allow a comparison.

\section{Maternal blood transfusion}

One study ${ }^{20}$ found no significant differences before/after OU closure (cOR $0.82,95 \%$ CI 0.55 to 1.21 ). The incidence of the outcome was low ( $<1 \%$ of women).

\section{Neonatal outcomes}

\section{Stillbirth (SB) (overall or intrapartum)}

Three studies examined the impact of OU closure on SB. One unpublished UK study (East Lancashire Hospitals NHS Trust, unpublished data 2017) showed a statistically significant reduction in SB over the period after the amalgamation of two units (cOR 0.61, 95\% CI 0.47 to 0.78 ). Similar findings were seen in one study from Canada ${ }^{22}$ during post closure years (COR $0.80,95 \%$ CI 0.67 to 0.96). A third study from the $\mathrm{UK}^{16}$ found no difference in $\mathrm{SB}$ rates after $\mathrm{OU}$ closure.

\section{Neonatal mortality (NM)}

Three studies reported this outcome. Two studies from the $\mathrm{UK}^{16}$ and (East Lancashire Hospitals NHS Trust, unpublished data 2017) showed no statistically significant difference in the rate of $\mathrm{NM}$ in the years after OU closure (cOR $1.33,95 \%$ CI 0.81 to 2.17 ; cOR $0.80,95 \%$ CI 0.29 to 2.26). A study from Norway ${ }^{17}$ also reported no difference (no OR presented).

\section{Perinatal mortality (PM)}

Two studies reported this outcome. In a study from Norway, ${ }^{7} \mathrm{PM}$ was significantly lower following OU closure (cOR $0.76,95 \%$ CI 0.58 to 0.98 ). A Canadian study ${ }^{22}$ also reported a significant reduction in PM after OU closure (cOR $0.75,95 \%$ CI 0.64 to 0.87 ).

\section{Infant mortality (IM)}

One study ${ }^{17}$ reported this outcome, IM rates were 'not statistically elevated' after the closure of thirteen hospitals in Norway. 


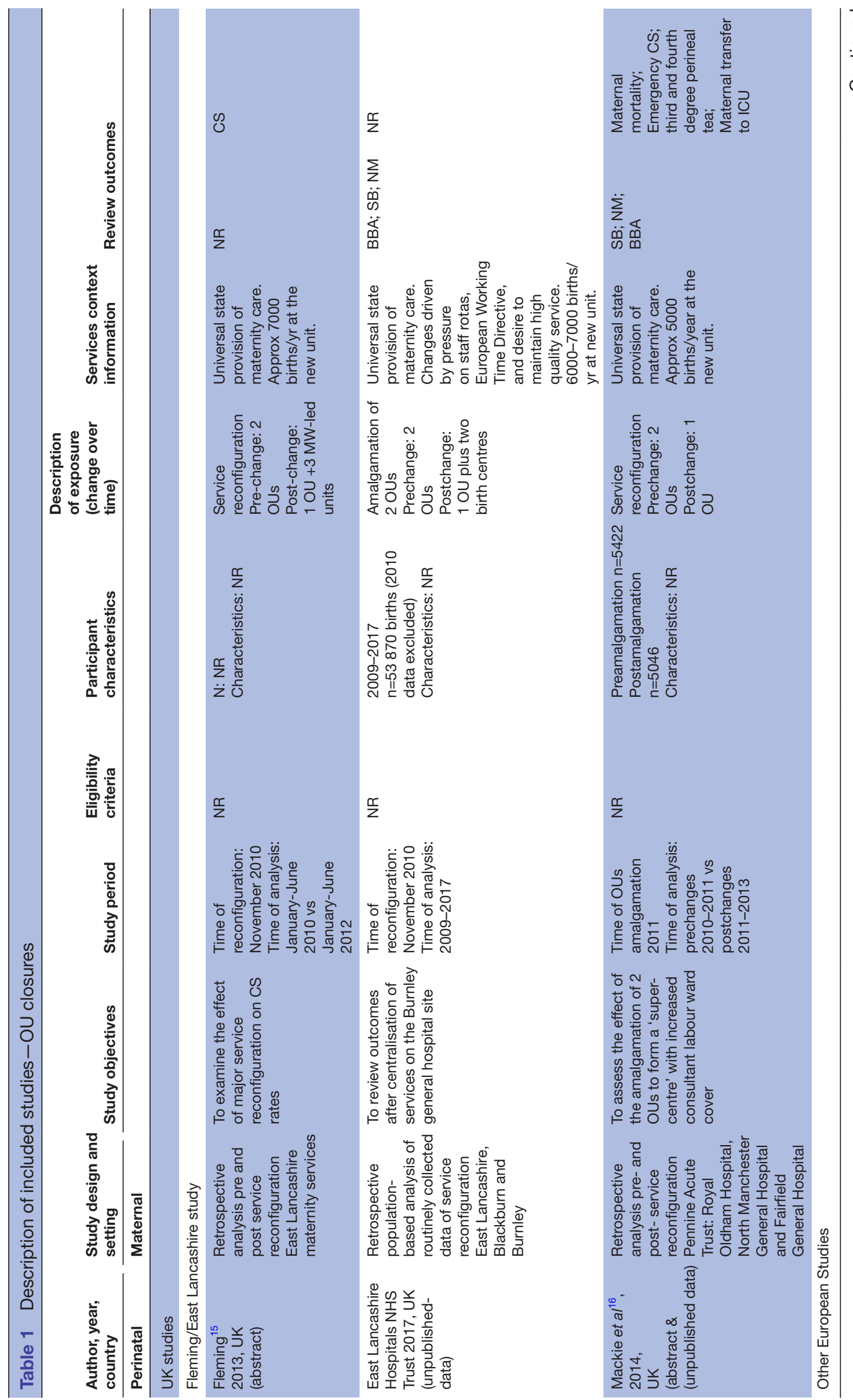




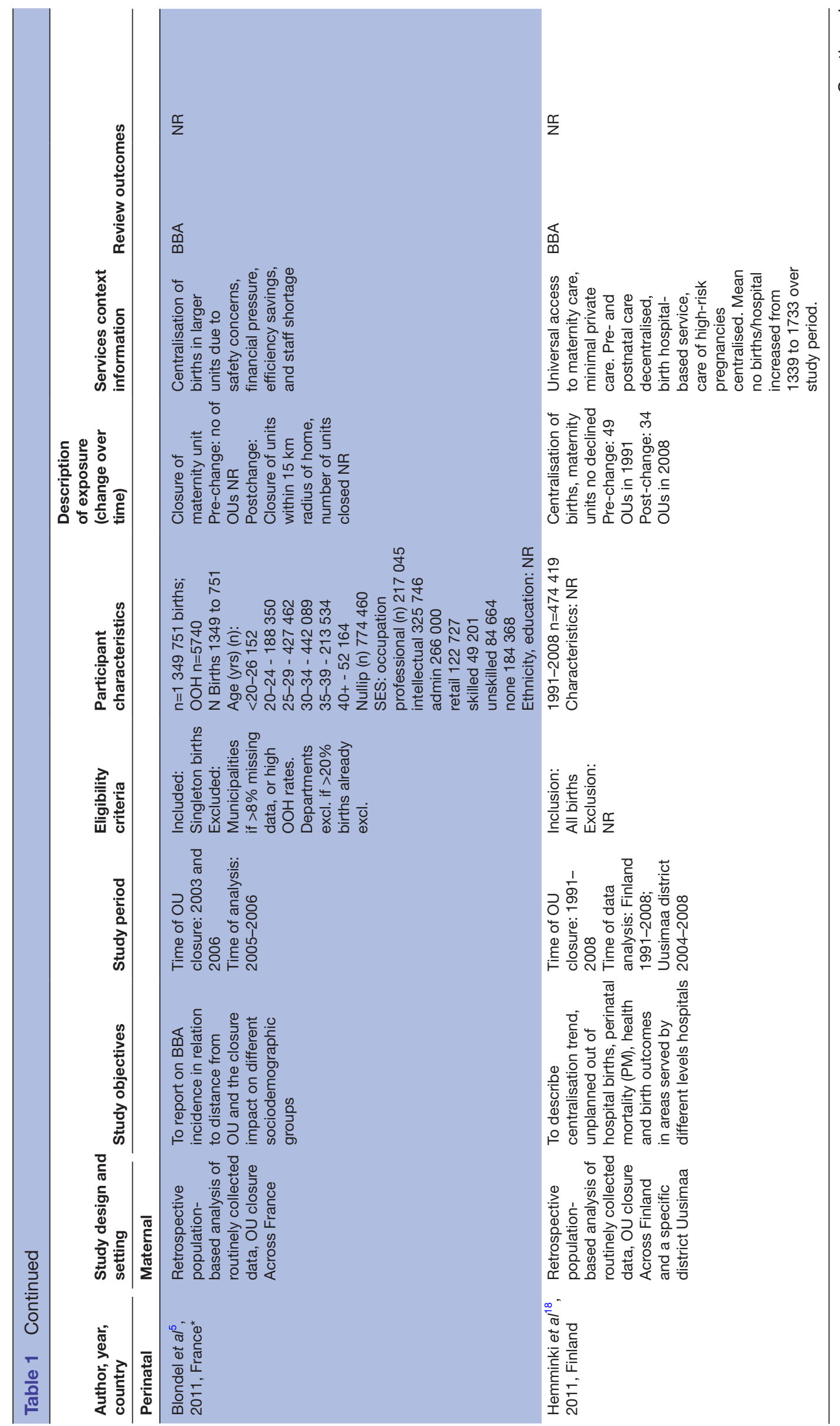




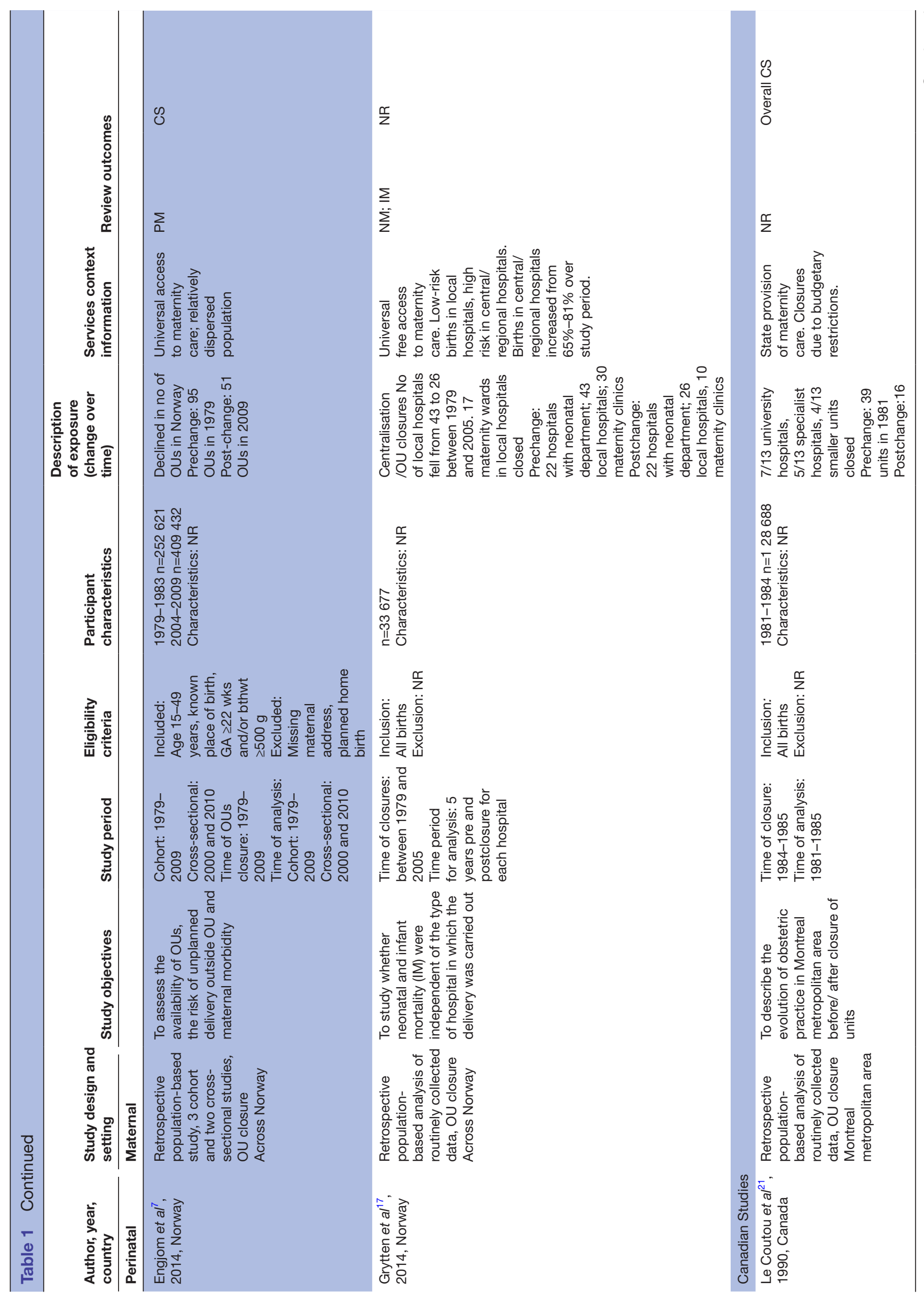



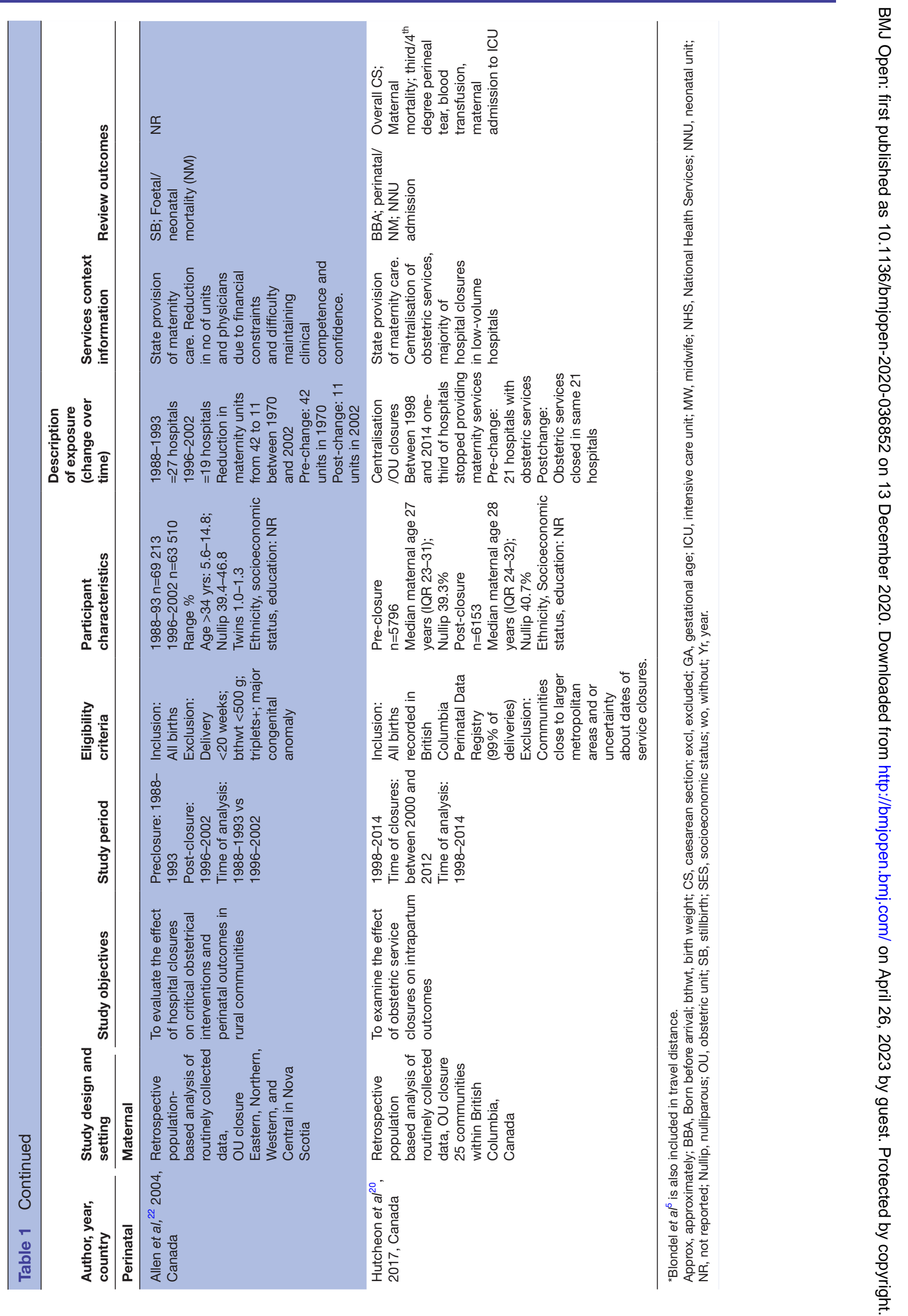

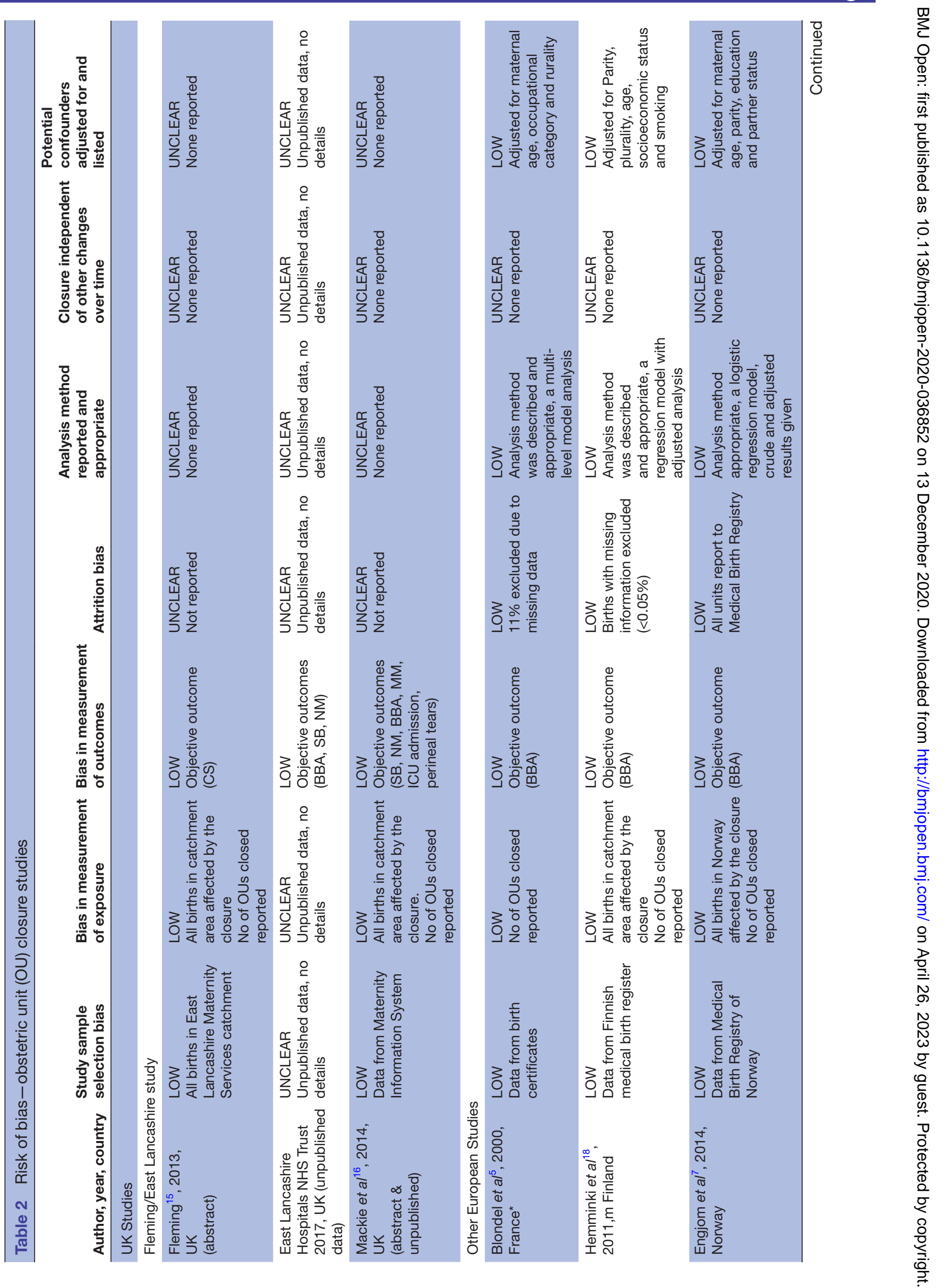


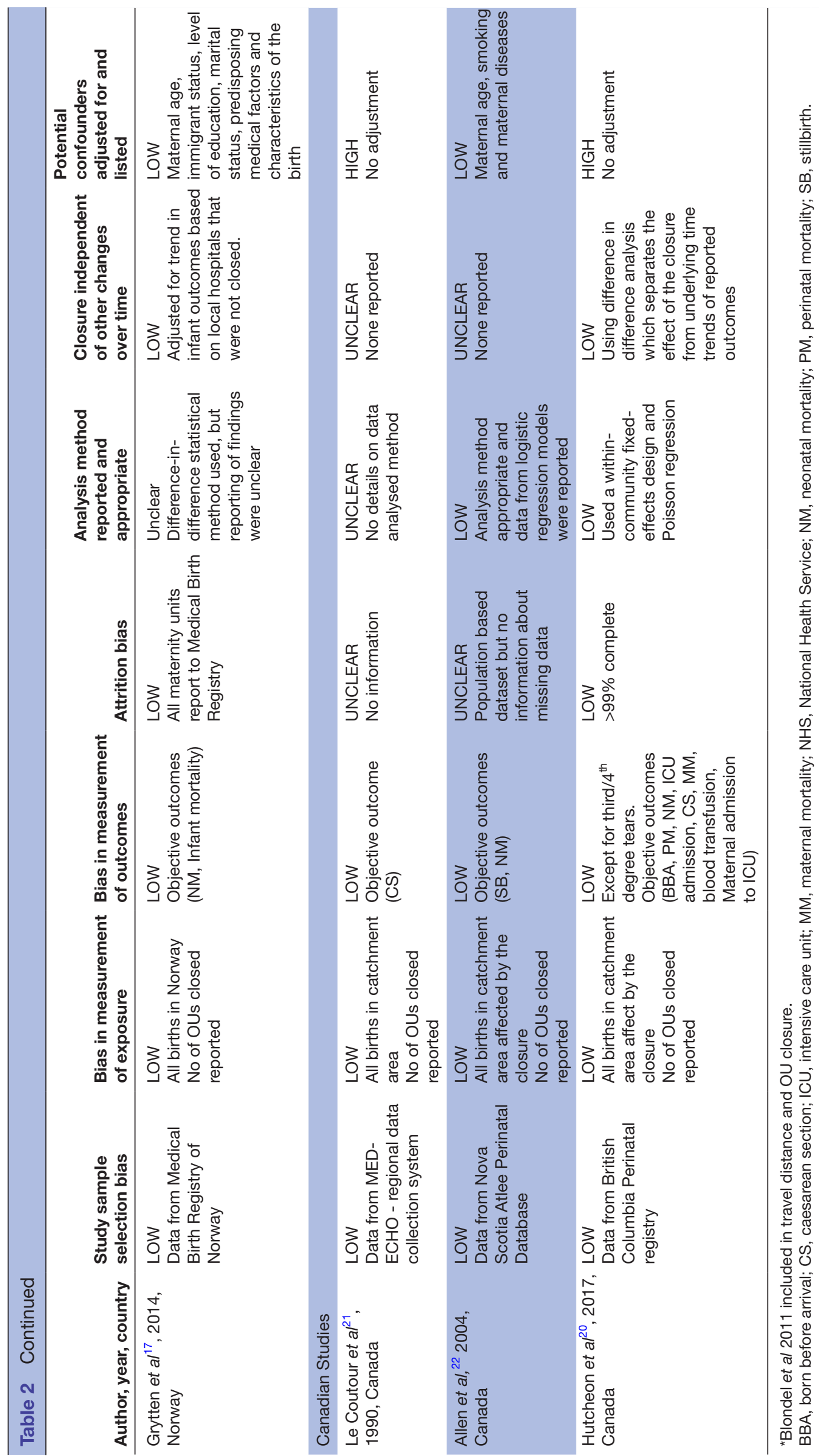




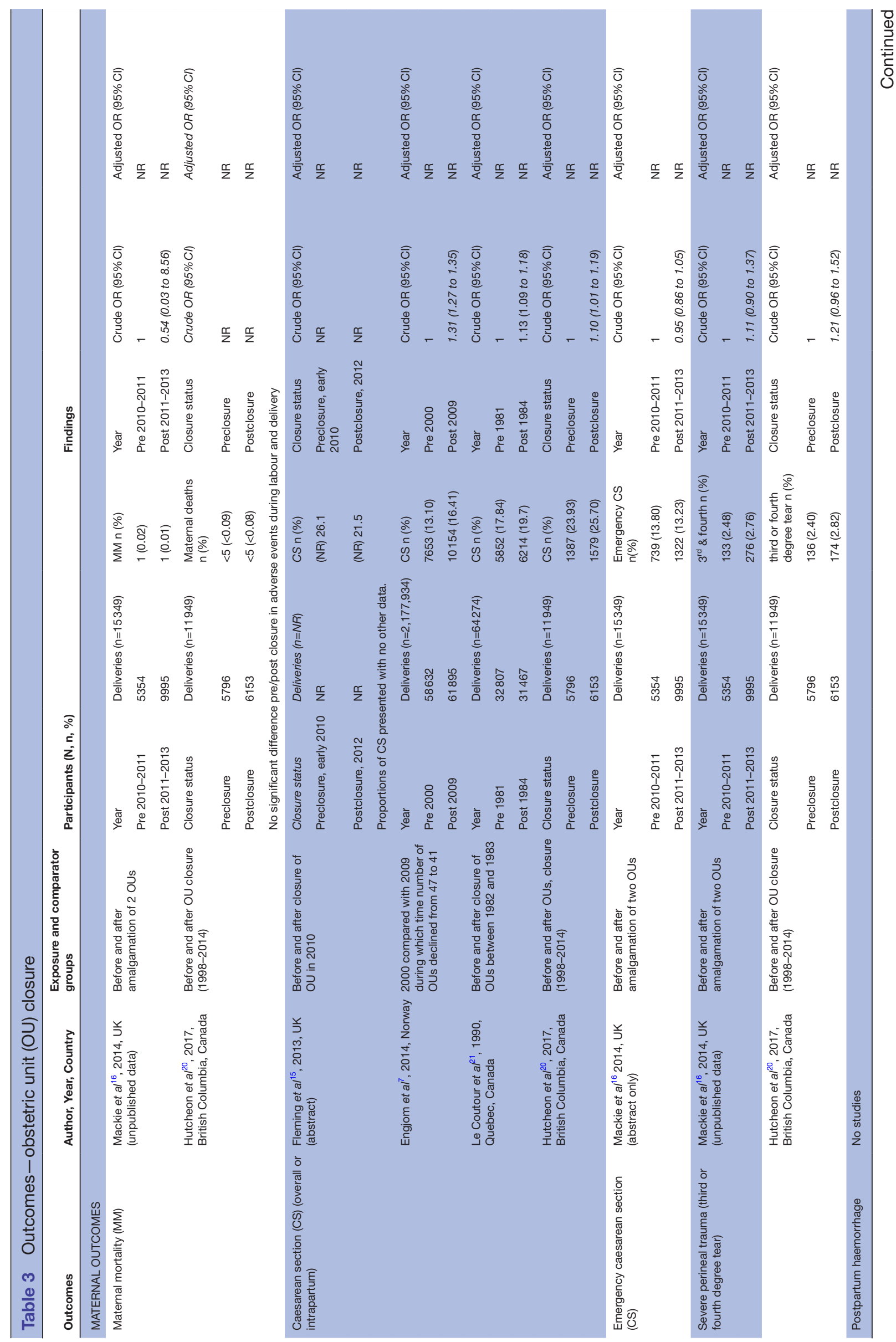




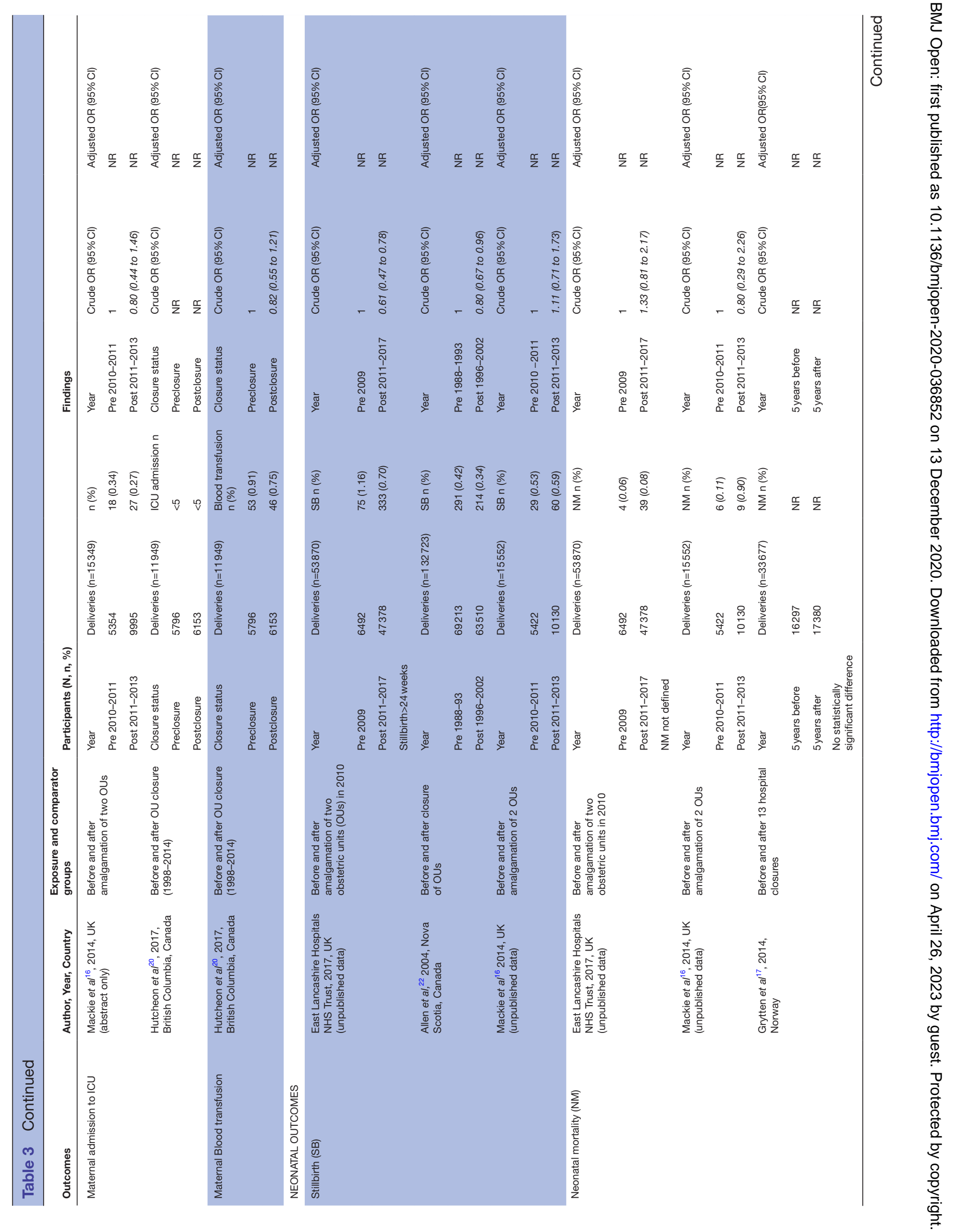




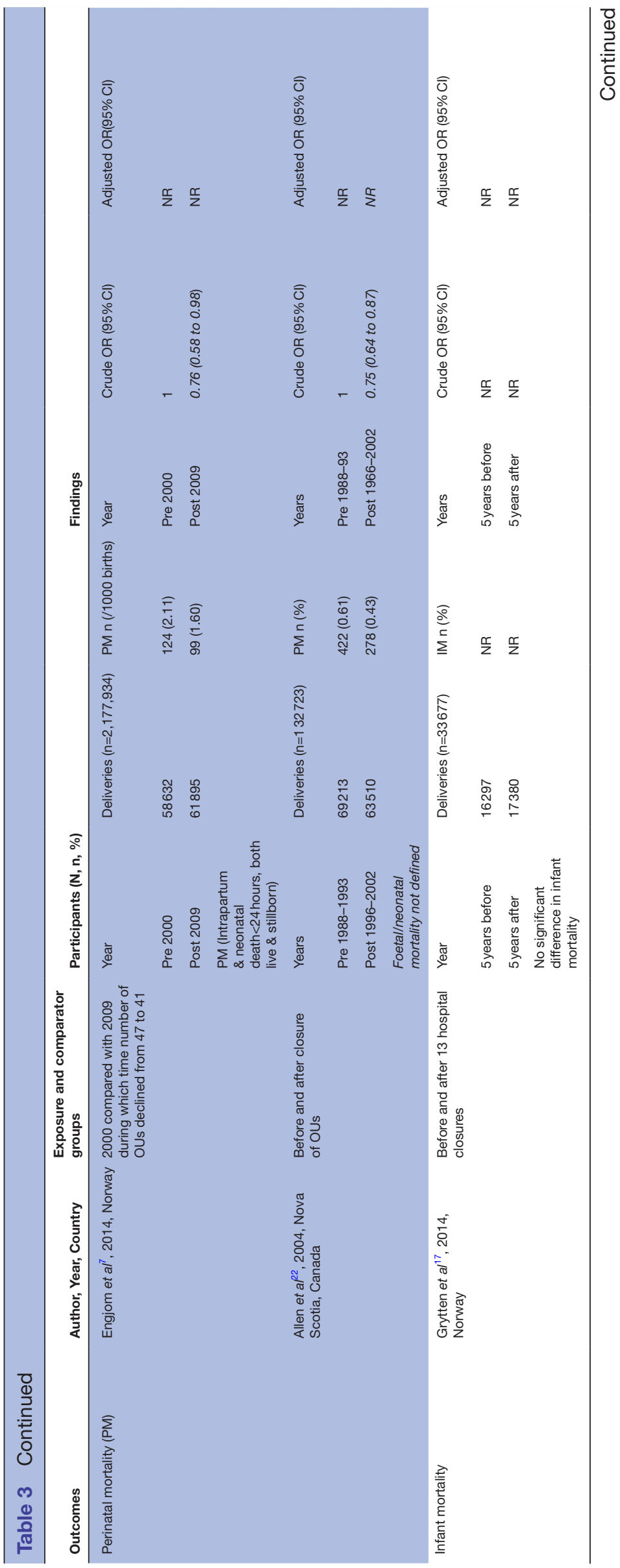

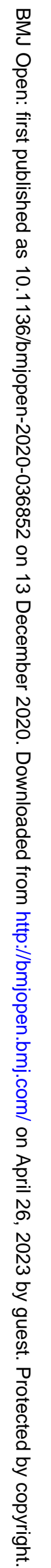




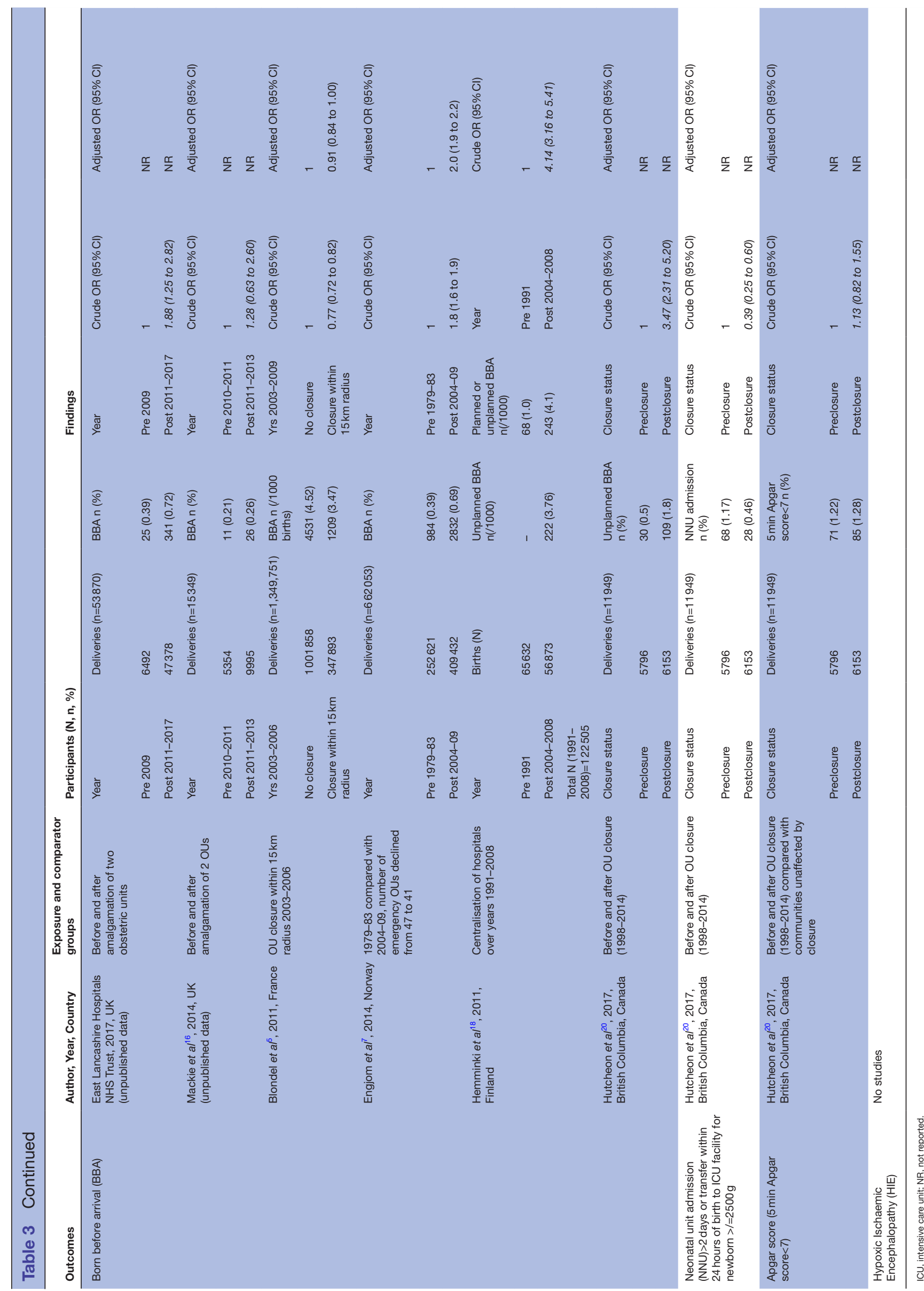




\section{Born before arrival (BBA)}

Six studies reported this outcome, with four suggesting a statistically significant increase in BBA following OU closure. Data from East Lancashire Hospitals NHS Trust (East Lancashire Hospitals NHS Trust, unpublished data 2017) showed the BBA rate almost doubled over the 10 -year period (cOR 1.88, 95\% CI 1.25 to 2.82). Studies from Norway ${ }^{7}$ and Finland ${ }^{18}$ also found that the BBA rate increased over a similar period (cOR 1.8, 95\% CI 1.6 to 1.9 and cOR $4.14,95 \%$ CI 3.16 to 5.41 , respectively). A Canadian study ${ }^{20}$ found that the BBA rate trebled over a 16-year period (cOR 3.47, 95\% CI 2.31 to 5.20 ). One UK study ${ }^{16}$ found no statistically significant change (cOR 1.28, $95 \%$ CI 0.63 to 2.60 ) and in one French study, ${ }^{5}$ there was weak evidence of a small reduction in the adjusted risk of BBA in communities affected by OU closure (adjOR 0.91, $95 \%$ CI 0.84 to 1.00$)$.

\section{Neonatal unit (NNU) admission}

One Canadian study ${ }^{20}$ suggested a significant reduction in NNU admission following OU closure (cOR 0.39, 95\% CI 0.25 to 0.60 ).

\section{Apgar score}

One Canadian study ${ }^{20}$ found no statistically difference in 5 min Apgar score of less than 7 before and after OU closure (cOR $1.13,95 \%$ CI 0.82 to 1.55 ).

\section{Hypoxic-ischaemic encephalopathy (HIE)}

No studies reported this outcome.

\section{Evidence from travel distance studies \\ Description of included studies}

Seven studies described the effect of travel distance to the nearest $\mathrm{OU}$ on maternal and neonatal outcomes (table 4). All were published in full between 1991 and 2015. The earliest study ${ }^{23}$ was conducted in the UK, three more recent studies were conducted in France, ${ }^{52425}$ and one each in Norway, ${ }^{26}$ Finland $^{27}$ and Canada. ${ }^{28}$ Four were retrospective population-based cohort studies, and three were case-control studies. The eligibility criteria varied across studies. Pasquier $e t a l^{24}$ included a group with special needs in the form of babies with congenital malformations. Only singleton live births were included in two studies. ${ }^{528}$

Travel distance was estimated using geographical mapping software in all studies. However, only three studies $^{524} 27$ measured the actual distance from women's homes to the nearest OU. In two studies ${ }^{2528}$ a central geographical point for the postal code or municipality was used to estimate distances and in one study the distance was self-reported. ${ }^{26}$ Additionally, the studies differed regarding their distance categories, which ranged from 2 to $150 \mathrm{~km}$ (table 4).

\section{Risk of bias assessment}

The main risk of bias concerns related to the measurement of exposure, as three studies ${ }^{25} 2628$ did not calculate the distance from the woman's home but used a central point instead or self-reported distance (table 5). Another study $^{23}$ measured distance between women's homes and hospital using a straight line. Further risk of bias related to a lack of comparability between study groups in the three case-control studies, ${ }^{23} 2627$ a lack of adjustment for confounders in two studies and missing data in two studies. ${ }^{2627}$ There were no risk of bias concerns relating to sample selection in the cohort studies or outcome measurement.

\section{Findings}

Maternal outcomes

\section{Maternal mortality (MM)}

In one case-control study from Finland, ${ }^{27}$ no maternal deaths were reported in either group (table 6).

\section{Caesarean section (CS) (overall or intrapartum)}

One study from Canada ${ }^{28}$ found no statistically significant differences in CS rates with increasing distance $(<50$ k, 50-150 k, >150 k) based on both crude and adjusted results.

\section{Emergency CS}

The same study from Canada ${ }^{28}$ reported no significant difference in emergency CS rates between women living at different distances from an OU based on cORs.

\section{Severe perineal trauma}

No studies reported this outcome.

Postpartum haemorrhage (PPH)

No studies reported this outcome.

\section{Maternal admission to ICU}

No studies reported this outcome.

\section{Maternal blood transfusion}

No studies reported this outcome.

\section{Neonatal outcomes}

Stillbirth (SB) (overall or intrapartum)

Two cohort studies ${ }^{25} 28$ reported this outcome. A Canadian study ${ }^{28}$ included births to women aged over 35 years who lived $<50 \mathrm{~km}, 50-150 \mathrm{~km}$ and $>150 \mathrm{~km}$ from the OU. SB rates did not change by distance category in the adjusted analysis. A French study ${ }^{25}$ reported SB rates at different distances $(<5 \mathrm{~km}, 5-15,15-30,30-44,45+\mathrm{km})$ from mother's municipality of residence to the closest OU. The cORs showed women living at 30-44, 15-29 and $5-14 \mathrm{~km}$ from an OU had a statistically significantly lower rate of $\mathrm{SB}$ compared with women living $<5 \mathrm{~km}$ from an OU (5-14 km vs $<5 \mathrm{~km}$, cOR $0.87,95 \%$ CI 0.85 to 0.90 ; $15-29 \mathrm{~km}$ vs $<5 \mathrm{~km}$; cOR 0.85 , 95\% CI 0.82 to 0.88 ; $30-44$ $\mathrm{km}$ vs $<5 \mathrm{~km}$, cOR $0.85,95 \%$ CI 0.81 to 0.90$)$. The findings still hold for the adjusted analysis (limited data reported). However, neither the crude nor the adjusted analysis showed a significant difference in risk of SB for individuals resident $45+\mathrm{km}$ from an OU compared with $<5 \mathrm{~km}$. 


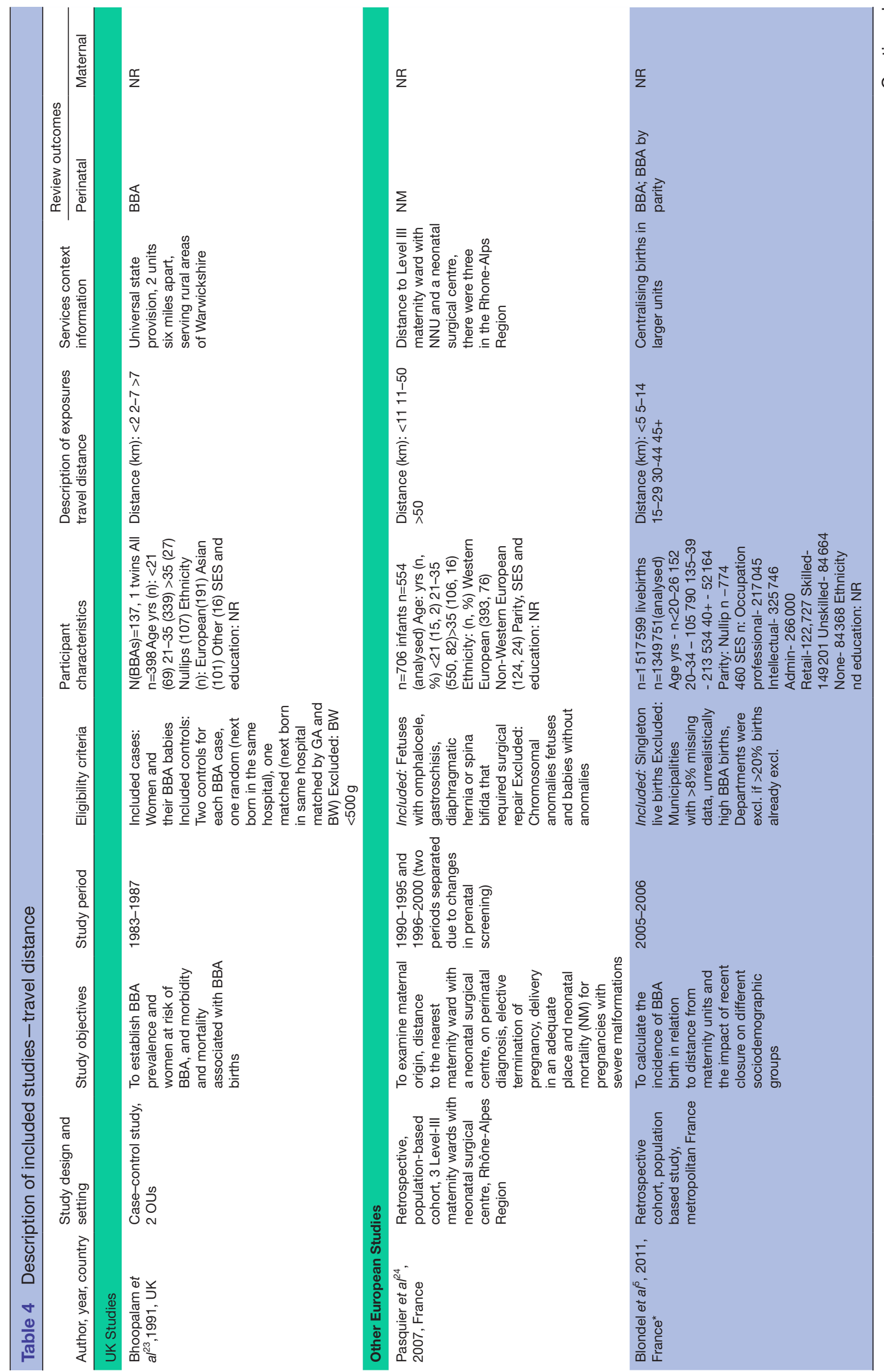




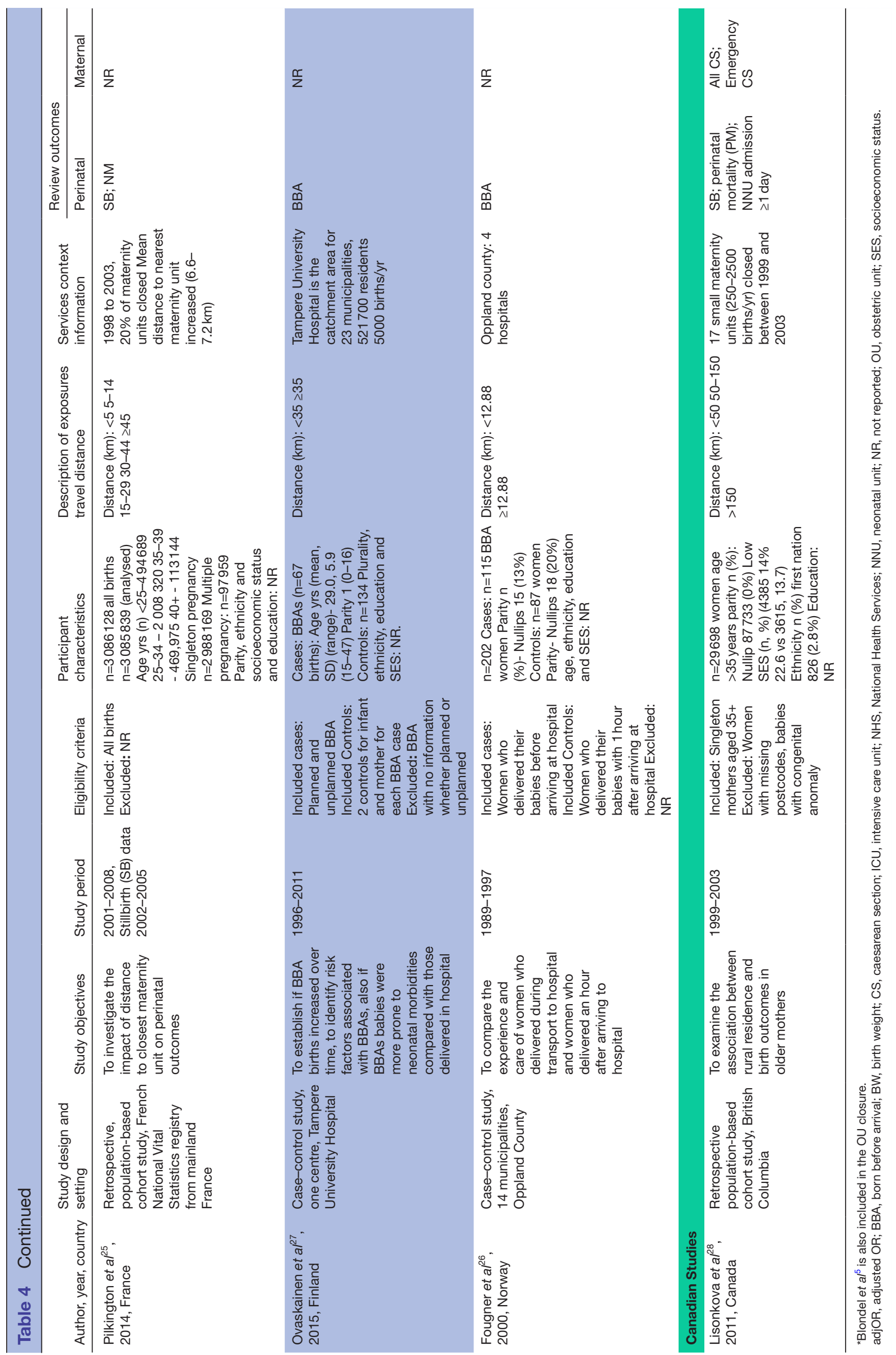




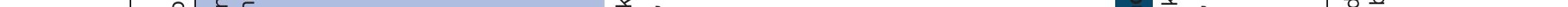




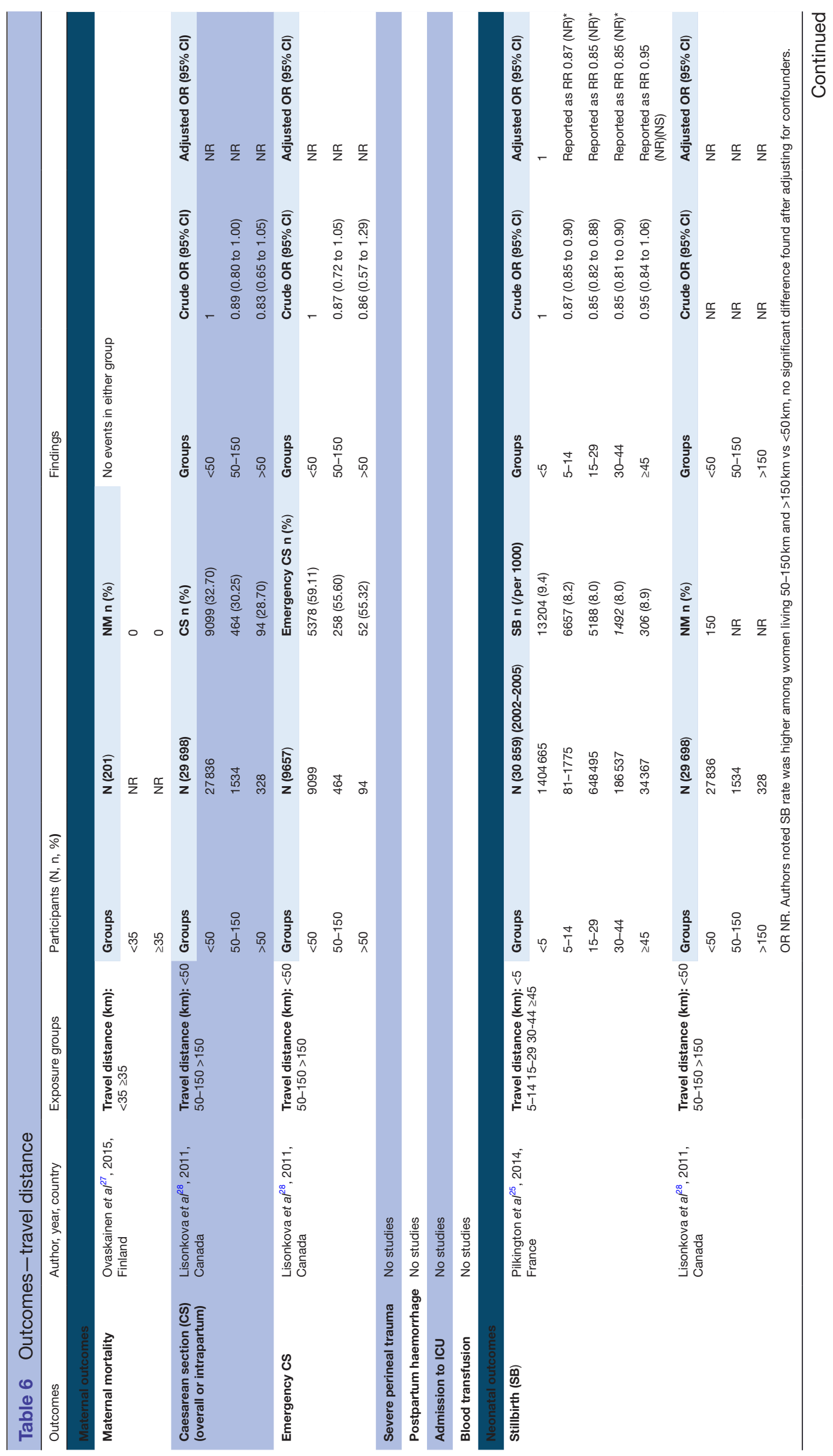

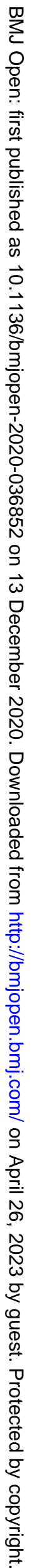




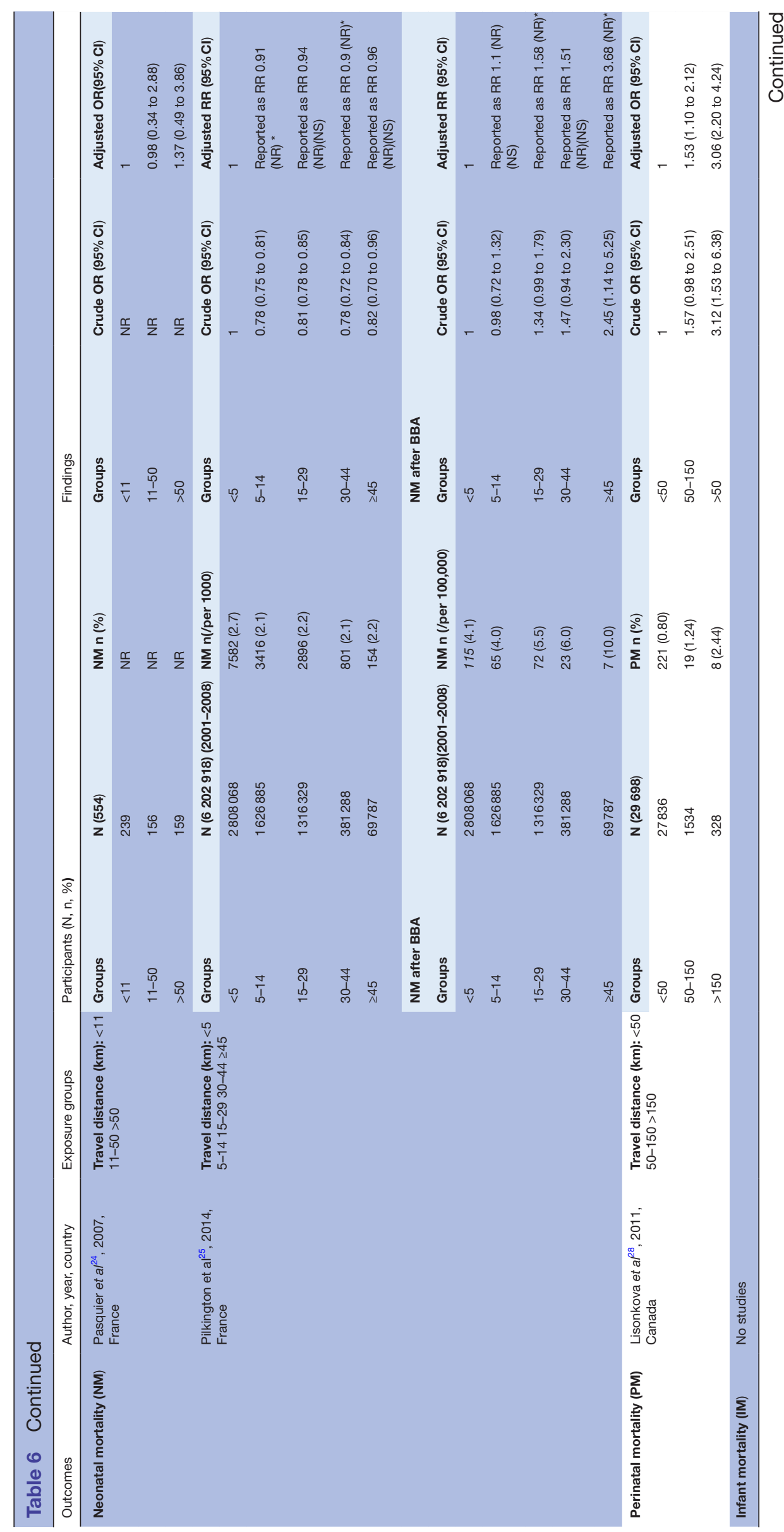

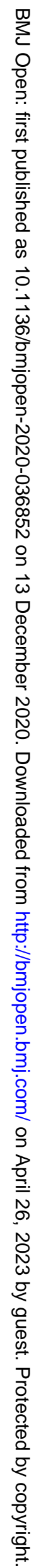




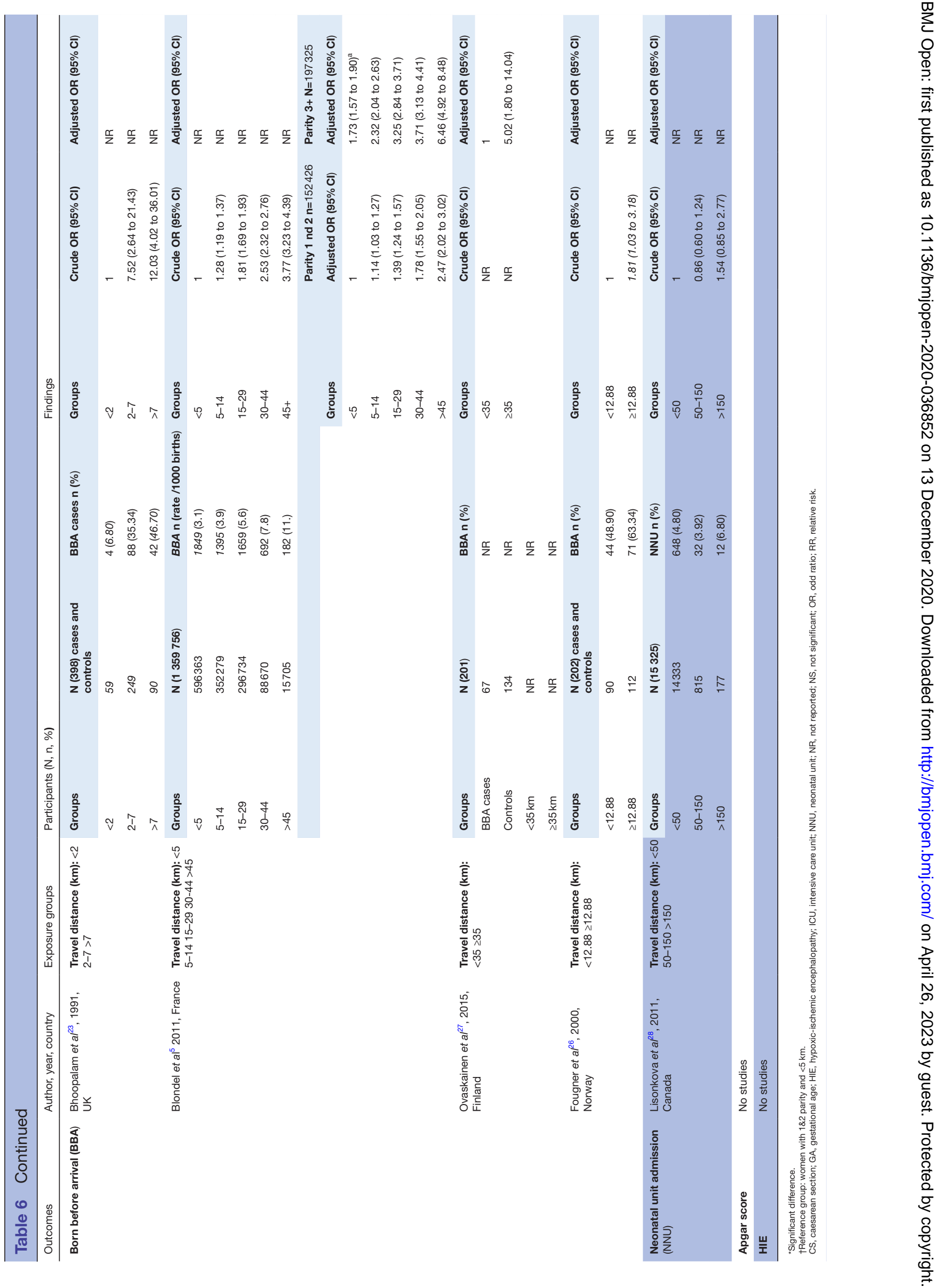




\section{Neonatal mortality (NM)}

Two French cohort studies ${ }^{24} 25$ reported this outcome. One study ${ }^{24}$ examined the distance from women's homes to the nearest OU with neonatal surgical facilities for 706 fetuses with severe malformations. Analyses adjusted for malformation type, number of malformations, amniotic fluid anomaly, previous anomaly in the family and parity showed no association between NM and distance $(<11$ $\mathrm{km}$ vs $11-50 \mathrm{~km}$, adjOR $=0.89,95 \%$ CI: $0.34,2.88 ;<11 \mathrm{~km}$ vs $>50 \mathrm{~km}$, adjOR=1.37, 95\% CI: $0.49,3.86)$. The other study ${ }^{25}$ included all births and found that NM rates were significantly higher for women living $<5 \mathrm{~km}$ compared with $5-44 \mathrm{~km}$ away from an $\mathrm{OU}^{25}(5-14 \mathrm{~km}$ vs $<5 \mathrm{~km}$, cOR $0.78,95 \%$ CI: $0.75,0.81 ; 15-29 \mathrm{~km}$ vs $<5 \mathrm{~km}$ cOR $0.81,95 \%$ CI: $0.78,0.85 ; 30-44 \mathrm{~km}$ vs $<5 \mathrm{~km}$, cOR 0.78 , $95 \%$ CI: $0.72,0.84 ; \geq 45 \mathrm{~km}$ vs $<5 \mathrm{~km}$, cOR $0.82,95 \%$ CI: $0.70,0.96)$. In this latter study, the NM of babies BBA was also explored. For the BBA group, there was a statistically significant increase in the risk of NM when women had to travel $45 \mathrm{~km}$ or more to an OU in comparison to $<5 \mathrm{~km}$ ( $\geq 45 \mathrm{~km}$ vs $<5 \mathrm{~km}$, cOR $2.45,95 \%$ CI 1.14 to 5.25 ).

\section{Perinatal mortality (NM)}

A study from Canada ${ }^{28}$ reported that PM risk increased with travel distance in an adjusted model $(<50 \mathrm{~km} \mathrm{v}$ $50-150 \mathrm{~km}$ adjOR $1.53,95 \%$ CI 1.1 to $2.12 ;<50 \mathrm{~km}>150$ $\mathrm{km}$ adjOR 3.06, 95\% CI 2.20 to 4.24$)$.

\section{Infant mortality (IM)}

No studies reported this outcome.

\section{Born before arrival (BBA)}

Three case-control studies, ${ }^{23} 2627$ and one cohort study ${ }^{5}$ reported this outcome. All four studies reported a significant increase in BBA rate with longer travel distance, although only two reported adjusted analyses. ${ }^{5} 27$ In the UK study ${ }^{23}$ the risk of BBA increased 12-fold for women living $>7 \mathrm{~km}$ from the OU compared with women living $<2 \mathrm{~km}$ away (cOR 12.5, 95\% CI 4.02 to 36.01 ). The risk of BBA increased significantly for women living $>13 \mathrm{~km}$ from an OU in a Norwegian study ${ }^{26}$ (cOR 1.81, 95\% CI 1.03 to 3.18 ). The Finnish study ${ }^{27}$ reported a fivefold increased risk of BBA for women living $>35 \mathrm{~km}$ from the OU compared with $<35 \mathrm{~km}$ (adjOR 5.02, 95\% CI 1.80 to 14.04).

In France, ${ }^{5}$ the rate of BBA significantly increased with longer distances and it tripled for all women living $45+$ $\mathrm{km}$ from the OU compared with women living $<5 \mathrm{~km}$ away (cOR 3.77, 95\% CI 3.23 to 4.39). The association persisted in an adjusted analysis which included women of parity three or higher and living $45+\mathrm{km}$ from the OU, who had a sixfold increased risk of BBA compared with women living $<5 \mathrm{~km}$ away and of parity one or two (adjOR $6.49,95 \%$ CI 4.92 to 8.48$)$.

Neonatal unit (NNU) admission

A study from Canada ${ }^{28}$ reported an increase in NNU admission for births to women living $>150 \mathrm{~km}$ from an OU compared with those living $<50 \mathrm{~km}$ away $(6.8 \%$ vs $4.8 \%)$.
Apgar score

No studies reported this outcome.

Hypoxic-ischaemic encephalopathy (HIE)

No studies reported this outcome.

Evidence from travel time studies

\section{Description of included studies}

Fifteen studies explored the impact of travel time from a woman's home to an OU (see table 7). Two studies (one reported as an abstract only) were conducted in the UK, ${ }^{29-31}$ three studies in France, ${ }^{32-34}$ three studies (reported in five articles) in the Netherlands, ${ }^{35-39}$ one study reported in two articles from Norway, ${ }^{40}$ five studies in Canada ${ }^{42-46}$ and one study in Japan. ${ }^{47}$

Eleven studies were of a retrospective cohort design, one was a prospective cohort study, ${ }^{39}$ one was a before-and-after $\operatorname{design}^{47}$ and two were case-control studies. ${ }^{33}{ }^{34}$ All the studies clearly stated the eligibility criteria. Only singleton births were included in five studies. ${ }^{30-32 ~ 35-38 ~} 42$ One study ${ }^{39}$ specifically enrolled women with postnatal haemorrhage after home birth, and one study ${ }^{42}$ focused on planned home birth regardless of the actual place of birth.

The studies were heterogeneous in their travel time intervals. With the exception of one study in Canada, ${ }^{42}$ longer time cut-off points were examined in studies from Norway, Japan and Canada compared with studies in other countries (all European). Travel duration was estimated using geographical mapping software in all studies. However, most studies estimated travel duration to and from central points within areas rather than actual addresses.

\section{Risk of bias assessment}

Risk of bias assessment and supported explanations for each of the risk of bias domains are presented in table 8 . With the exception of Stolp et al, ${ }^{39}$ sample selection and measurement of outcomes were considered to be at low risk of bias across all studies as such data were obtained from national databases and birth registries. The groups in the two case-control studies were appropriately selected and defined, however, the case and control groups were not comparable in both studies (eg, difference in antenatal care attendance and sociodemographics). Eight studies ${ }^{29} 34-42$ were considered at low risk of exposure measurement bias, as the women's actual place of residence was used to estimate travel time to nearest OU. The risk of attrition bias was low for the majority of the included studies. Similarly, analyses and adjustment for potential confounders were found to be appropriate in the majority of studies.

\section{Findings}

Maternal outcomes

Maternal mortality (MM):

No studies reported this outcome. 

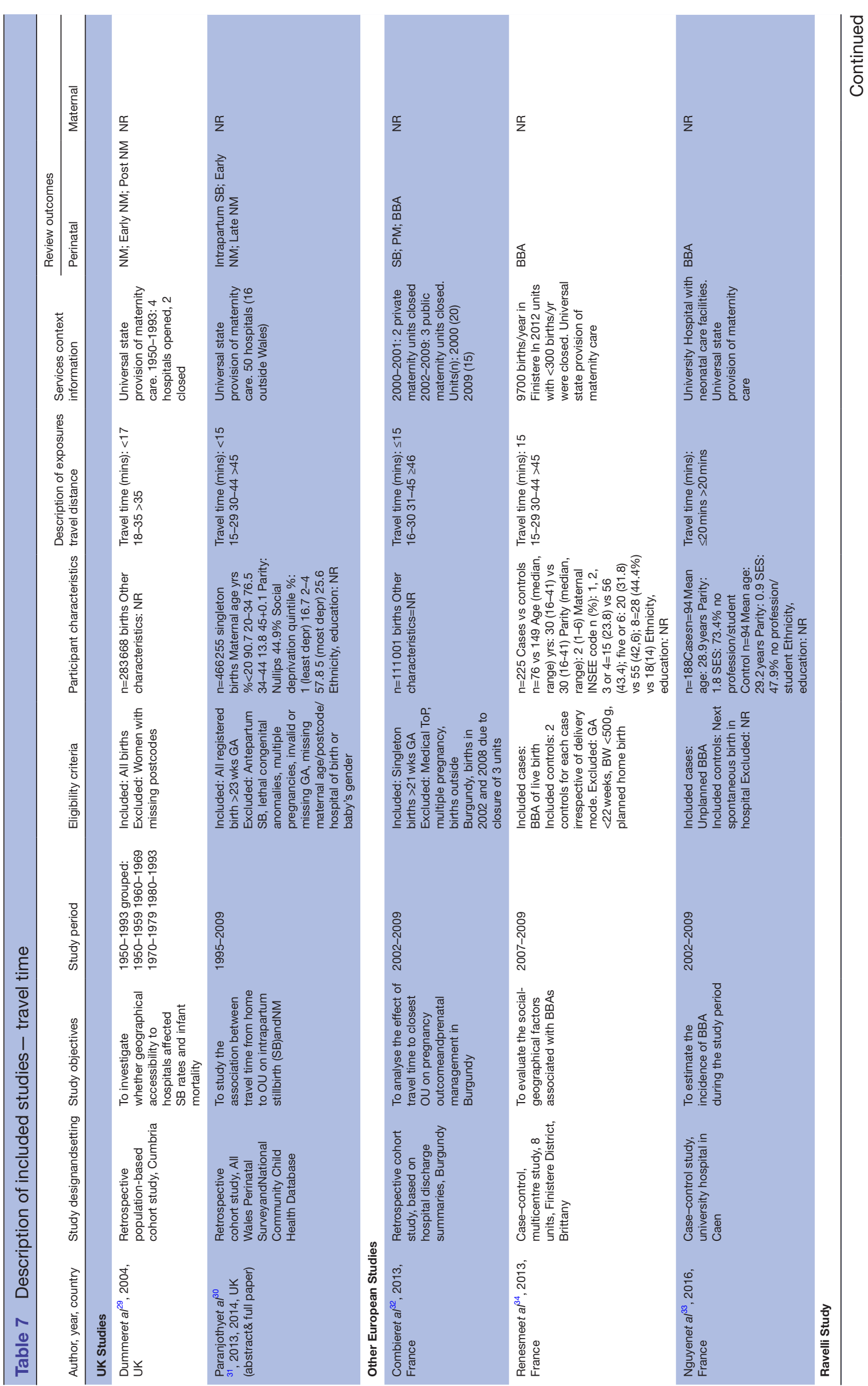

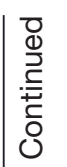

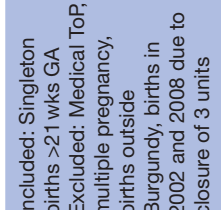

命

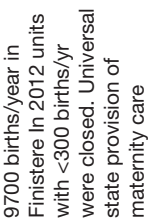

$\frac{\pi}{2}$

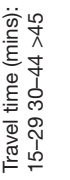

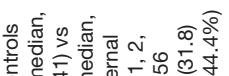

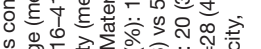

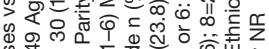

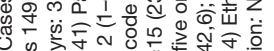

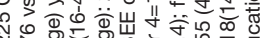

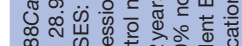

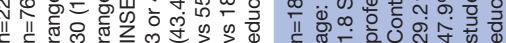

䒽
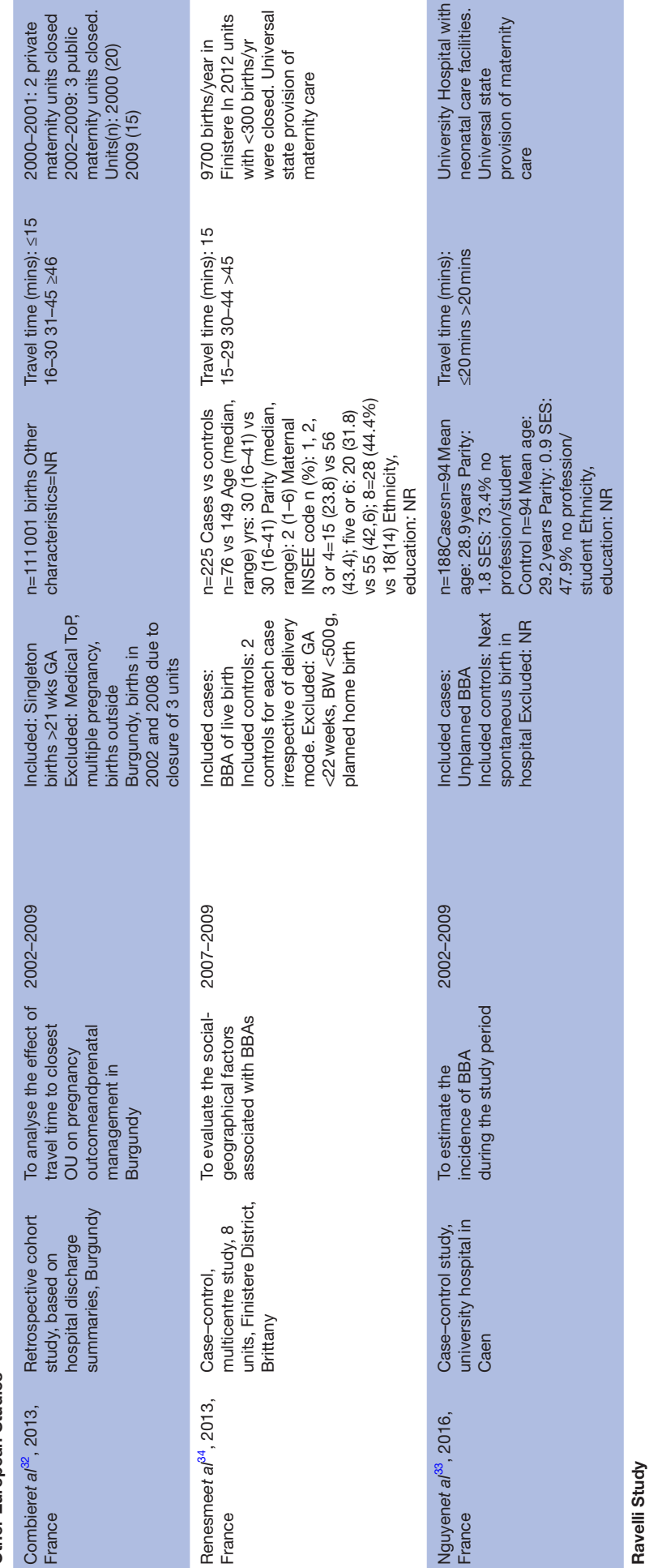

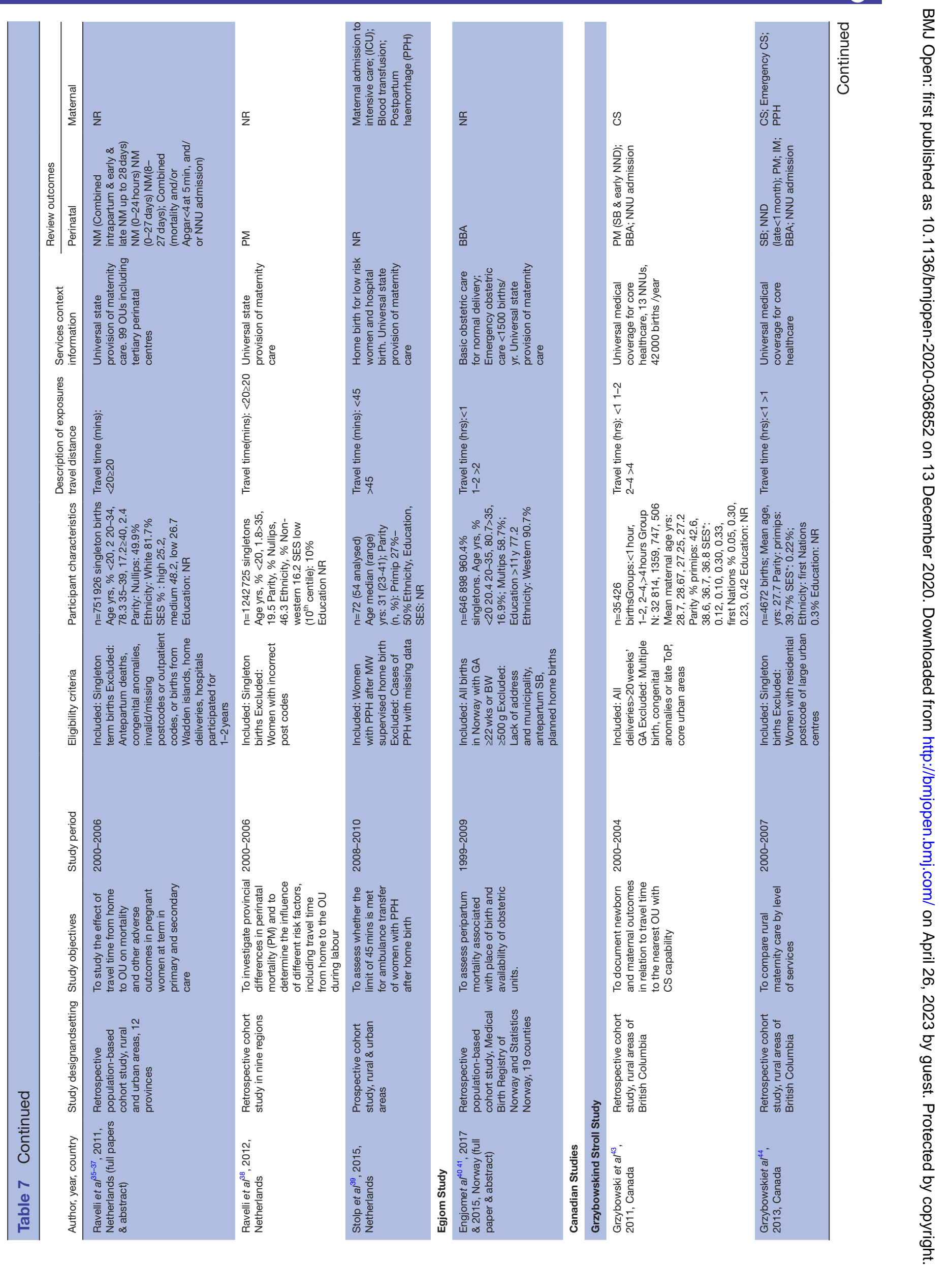


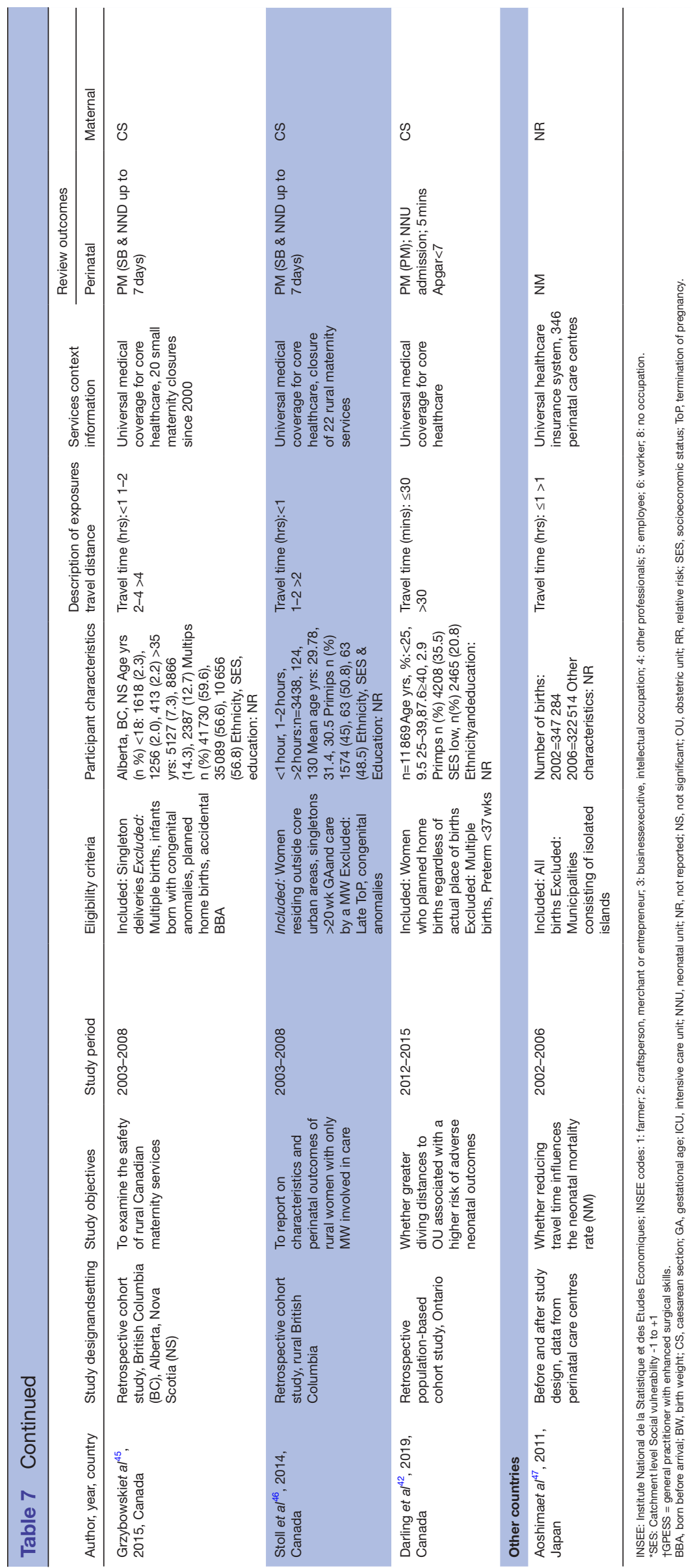




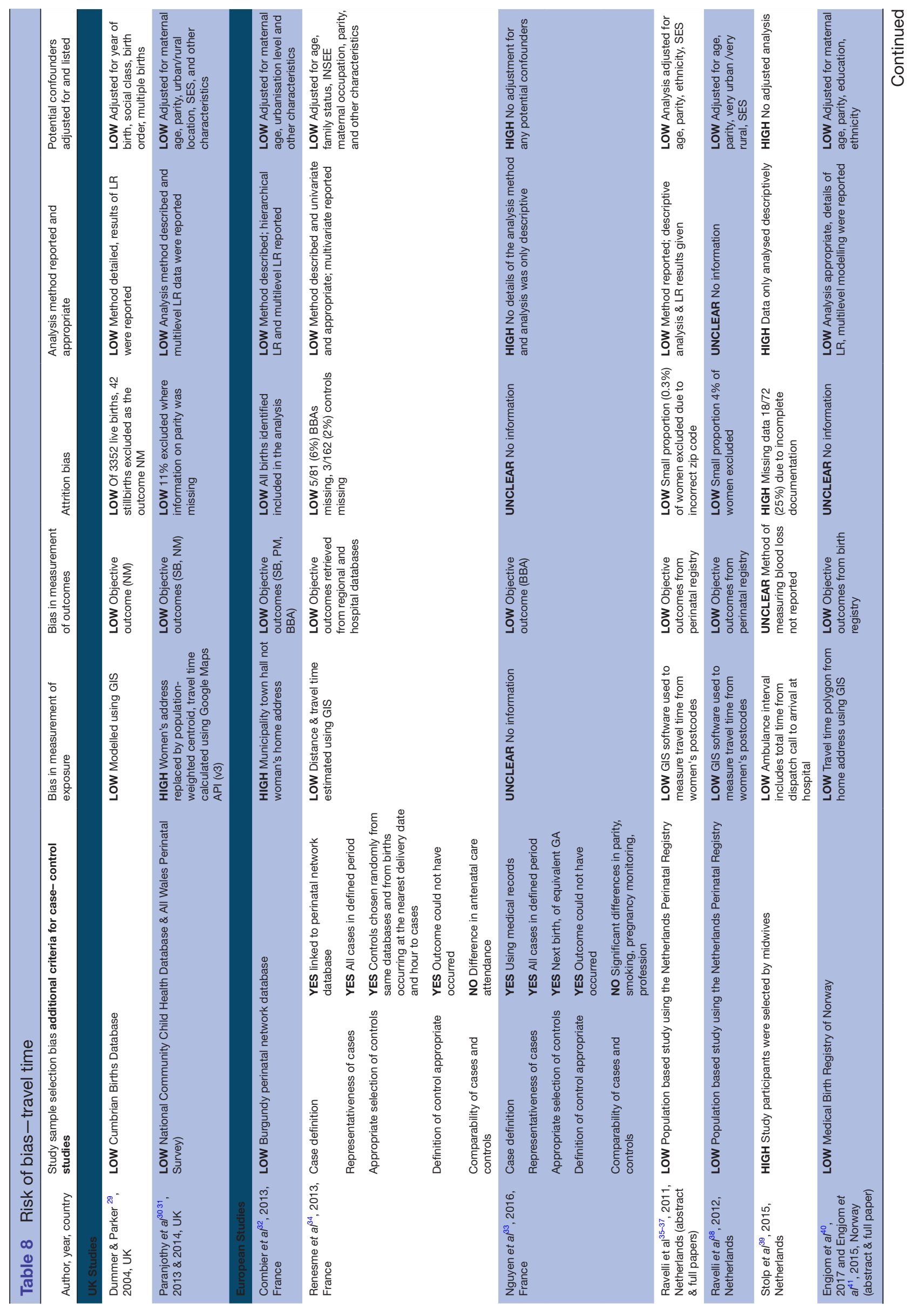

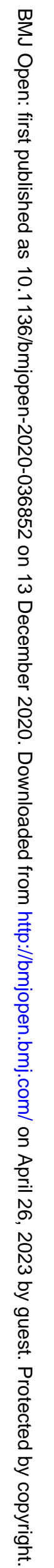




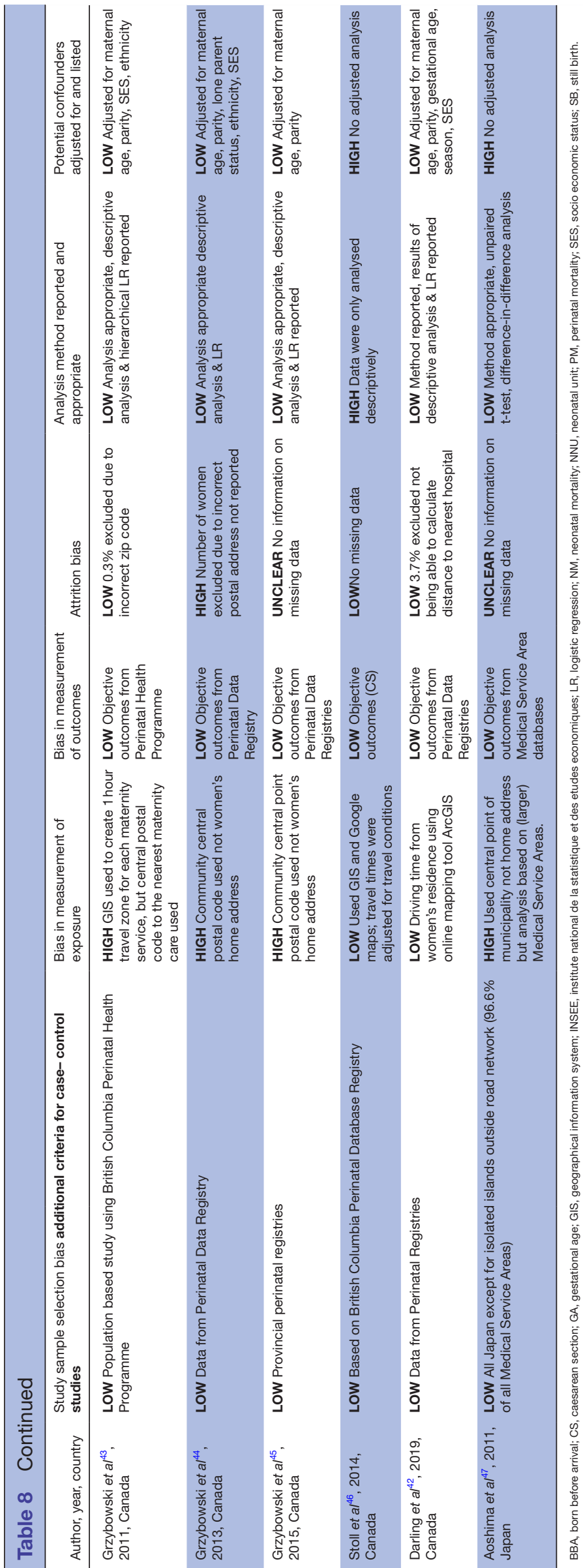


Caesarean section (CS) (overall, or intrapartum)

Five Canadian studies ${ }^{42-46}$ reported CS rates (table 9). Across three studies, ${ }^{43-45}$ cORs for CS rates were higher among women who lived closer to OUs with CS rates highest for women living less than 1 hour away compared with other categories (1-2 hours, $2-4$ hours and $>4$ hours). One study ${ }^{46}$ included women who had a midwife involved in their care, and found no significant differences in CS rates for women living 1-2 hours and more than 2 hours away compared with within 1 hour of an OU (1-2 vs $<1$ hour, cOR $1.23,95 \%$ CI 0.80 to 1.91 and $>2$ hours vs $<1$ hour, cOR $1.11,95 \%$ CI 0.71 to 1.72 ). A further study ${ }^{42}$ also showed a higher CS rate among women who planned a home birth and lived less than half an hour away from OU services (>30 $\min$ vs $\leq 30 \mathrm{~min}$, cOR $0.74,95 \%$ CI 0.59 to 0.92 ).

\section{Emergency CS}

Shorter travel time to an OU was associated with a statistically significant higher emergency CS rate in one Canadian study ${ }^{45}$ (>1 hour vs $<1$ hour, cOR $0.80,95 \%$ CI 0.75 to 0.86$)$.

\section{Severe perineal trauma}

No studies reported this outcome.

\section{Postpartum haemorrhage (PPH)}

One Canadian study found the risk of PPH was significantly higher for women who lived more than 1 hour away from obstetric services compared with women who lived less than 1 hour away ${ }^{44}(>1$ hour vs $<1$ hour, cOR $1.27,95 \%$ CI 1.13 to 1.43$)$.

\section{Maternal admission to ICU}

One study from the Netherlands ${ }^{39}$ involved women who had a PPH after midwifery-supervised home births and examined adverse maternal outcomes associated with travel time longer than $45 \mathrm{~min}$ to hospital. No difference was found in the number of women admitted to ICU who travelled more than $45 \mathrm{~min}$ compared with $<45$ travel time to hospital, but the numbers of events were low.

\section{Maternal blood transfusion}

One study from the Netherlands ${ }^{39}$ found no significant difference in the median number of units of blood transfused to women who travelled more than $45 \mathrm{~min}$ to an OU compared with $<45$ min travel time.

\section{Neonatal outcomes:}

\section{Stillbirth (SB) (overall or intrapartum)}

Three studies examined the association between increasing travel time and SB, one study each from the UK, ${ }^{30}{ }^{31}$ France $^{32}$ and Canada. ${ }^{44}$

In the UK study, ${ }^{30} 31$ there was no association between travel time and SB when analysing all women (adjOR 1.13, $95 \%$ CI 0.98 to 1.30 ). However, subgroup analyses showed a significant increase in the risk of SB with every $15 \mathrm{~min}$ increase in travel time to the OU for term pregnancies (adjOR 1.36, 95\% CI 1.17 to 1.59 ) and for nulliparous women (adjOR 1.21, 95\% CI 1.02 to 1.44 ). The other two studies $^{32} 44$ found no significant increase in the incidence of SB with increasing travel time.

\section{Neonatal mortality (NM)}

Five studies examined the association between travel time and NM, two from the UK, ${ }^{29-31}$ one from the Netherland, ${ }^{35-37}$ one from Canada ${ }^{44}$ and one from Japan. ${ }^{47}$

The adjusted analysis in one UK study ${ }^{29}$ showed no statistically significant association between NM and travel time. The adjusted analyses in the other UK study ${ }^{31}$ showed a significant increase in early and late NM, with every $15 \mathrm{~min}$ increase in travel time (adjOR 1.13, 95\% CI 1.07 to 1.20 ) and (adjOR $1.15,95 \%$ CI 1.05 to 1.26 ) respectively. Subgroup analysis for nulliparous women showed a statistically significant increased risk of early $\mathrm{NM}$ associated with every $15 \mathrm{~min}$ increase in travel time from home to the OU (adjOR 1.15, 95\% CI 1.06 to 1.25 ). For term births, late (but not early) NM increased significantly with every 15 min increase travel time from home to the OU (adjOR 1.34, 95\% CI 1.13 to 1.59 ).

In one study from the Netherlands, ${ }^{35}$ a travel time of 20 min or more was associated with a significant increase in the combined intrapartum, early and late $\mathrm{NM}^{35-37}(\geq 20$ $\min$ vs $<20 \mathrm{~min}$, adjOR $1.23,95 \%$ CI 1.07 to 1.41 ). No NM events were reported in the study from Canada. ${ }^{44}$ The study from Japan ${ }^{47}$ reported that following a median reduction in travel time from $67 \mathrm{~min}$ in 2002 to $39 \mathrm{~min}$ in 2006 that there was a decrease in NM rate from 1.67 to 1.28 , however, no further analyses were presented.

\section{Perinatal mortality (PM)}

Seven studies examined PM, one from France, ${ }^{32}$ one from the Netherlands ${ }^{38}$ and five from Canada. ${ }^{42-46}$ The French study ${ }^{32}$ found no significant association between increasing travel time to the nearest OU and PM based on unadjusted data. However, in the Dutch study a longer travel time (20 min or more) was significantly associated with higher $\mathrm{PM}^{38}(\geq 20$ min vs $<20$ min, adjOR $1.66,95 \%$ CI 1.59 to 1.74 ).

The Canadian studies also reported longer travel times to OUs being associated with an elevated risk of PM. A significant increase in PM was reported in women living more than 4 hours away from OUs compared with women living less than 1 hour ( $>4$ hours vs $<1$ hour adjOR 3.17, 95\% CI 1.45 to 6.95$).{ }^{43}$ However, findings from the same study suggested no significant increase for women living 1-2 hours and 2-4 hours from an OU compared with those living less than 1 hour from services. Similarly, the PM risk significantly increased in women who lived $>1$ hour from OUs in a further Canadian study ${ }^{44}$ (cOR 1.54, 95\% CI 1.09 to 2.17). When this was divided into different Canadian provinces, ${ }^{45}$ the rates of PM were highest in communities living more than 4 hours from an OU in comparison to less than 1 hour in British Colombia only (adjOR 2.84, 95\% CI 2.84 to 5.10). Stoll and Kornelsen, ${ }^{46}$ found that in women who received midwifery care only, PM was not statistically significantly different for women 


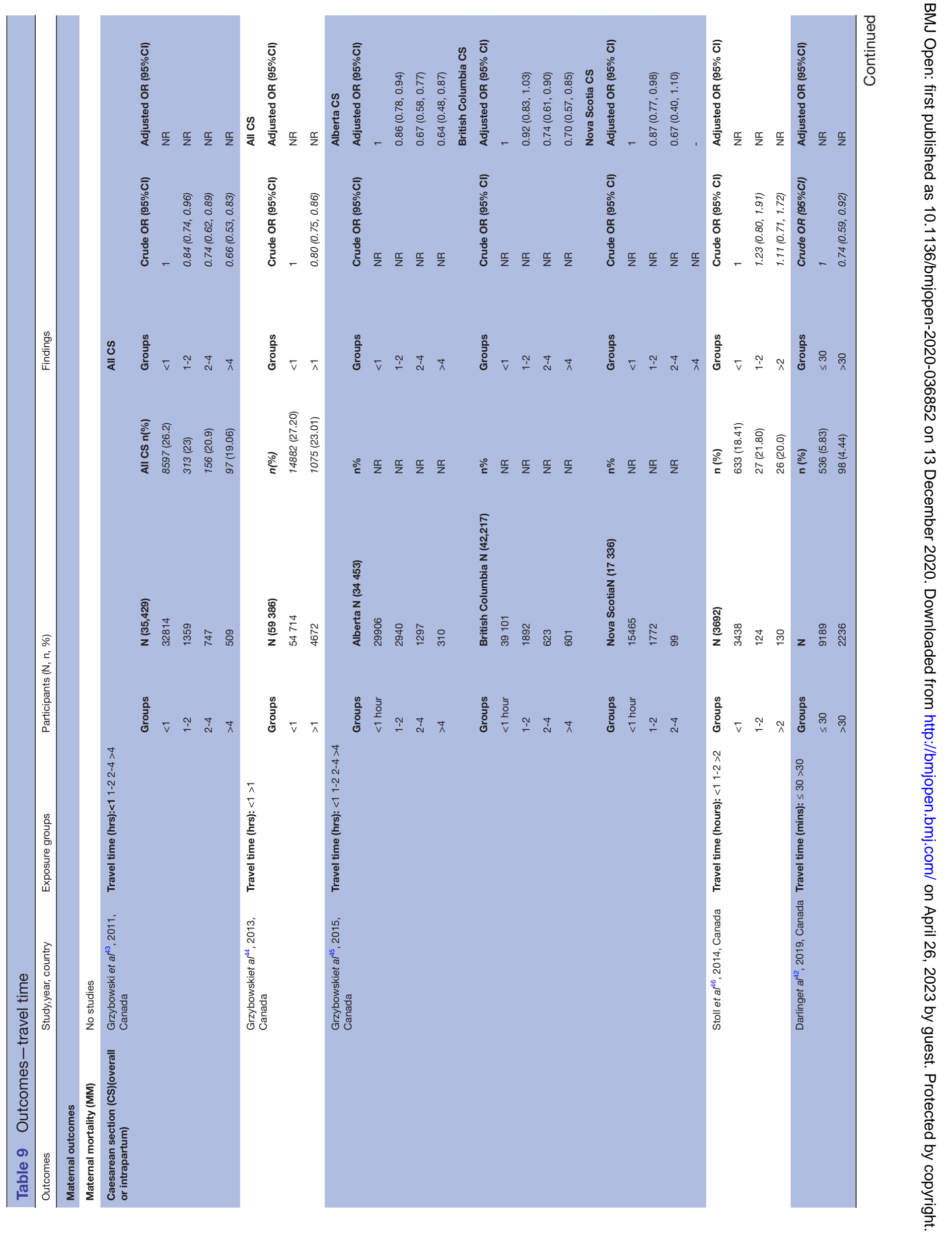




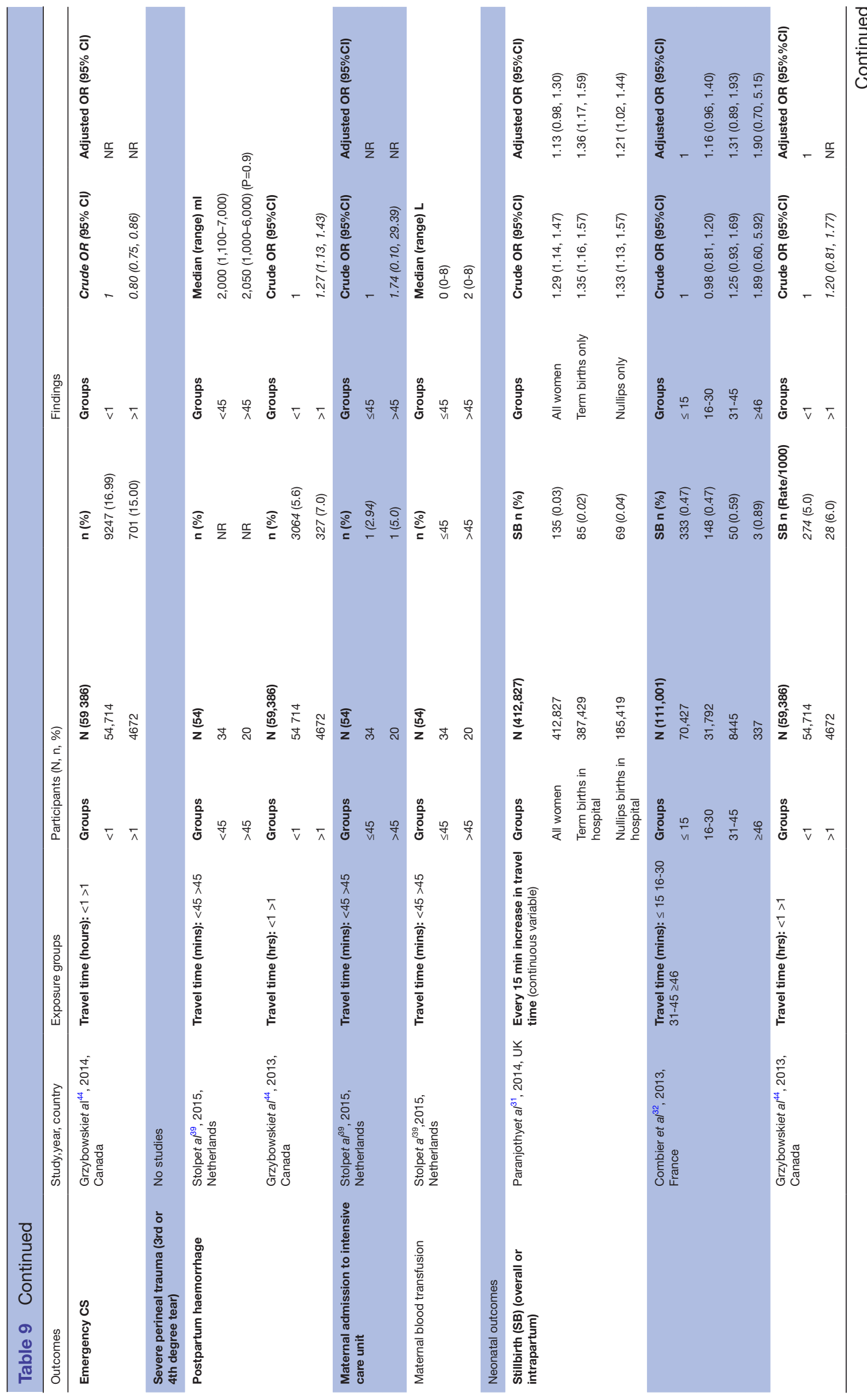




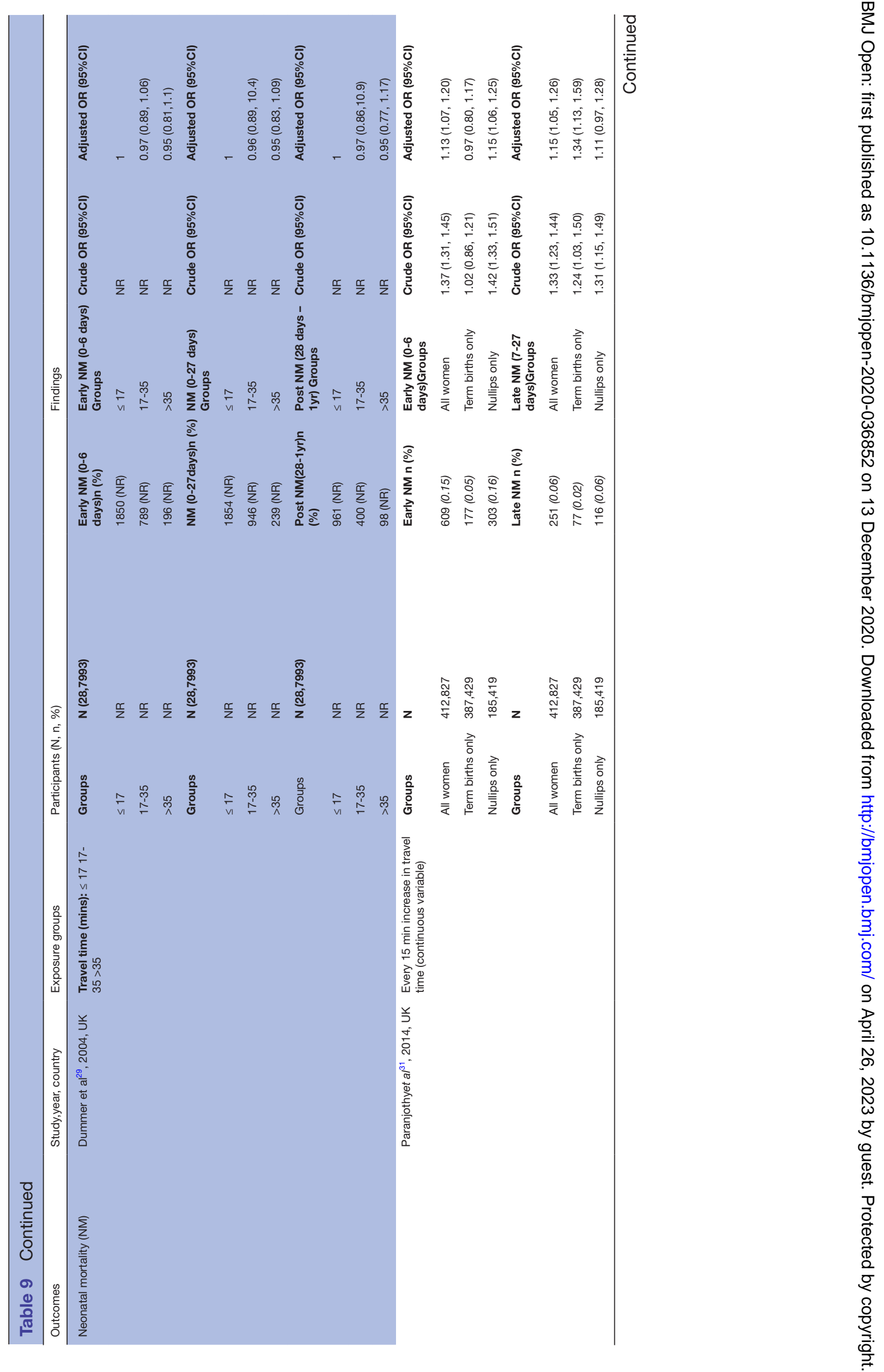




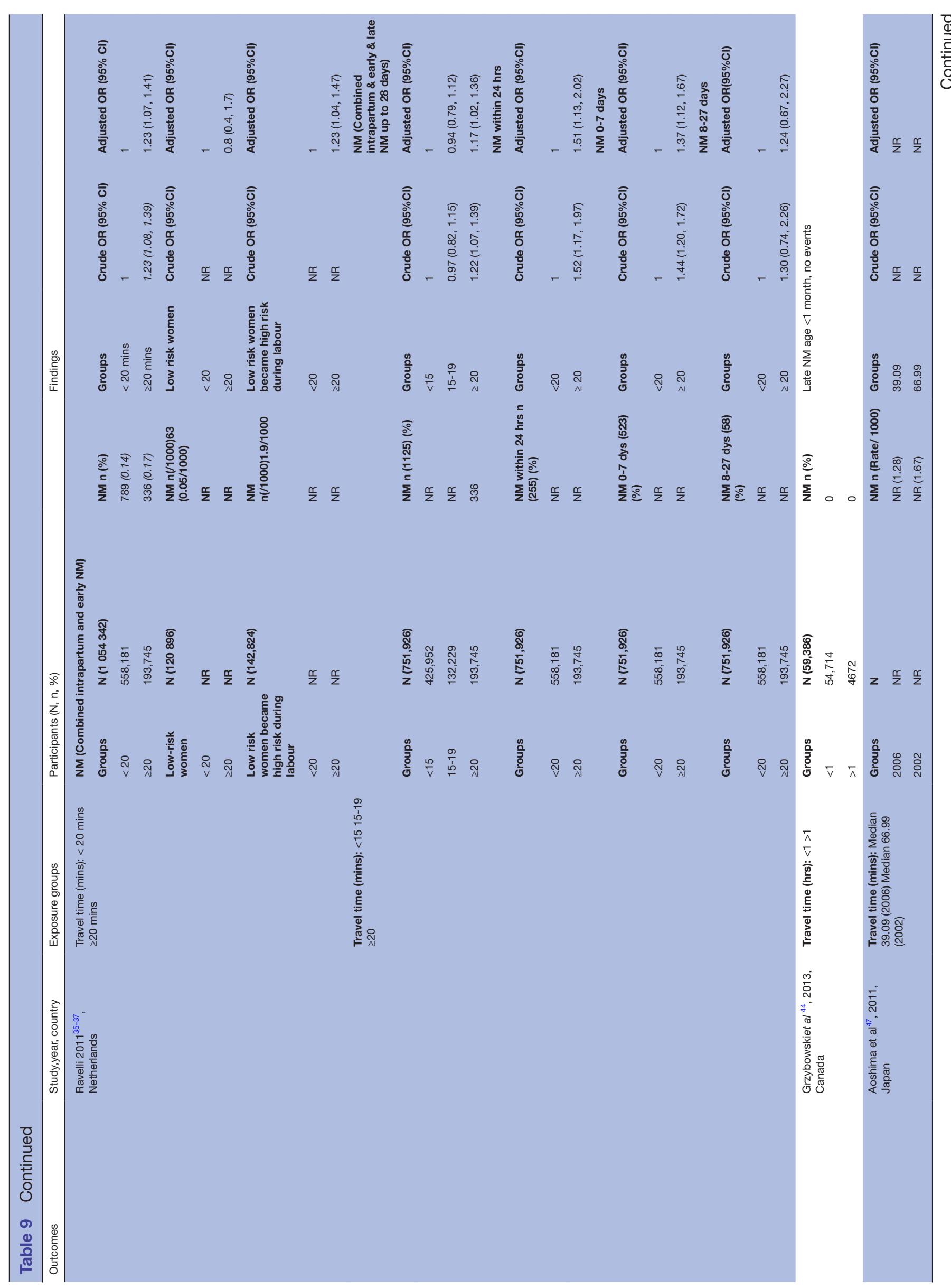




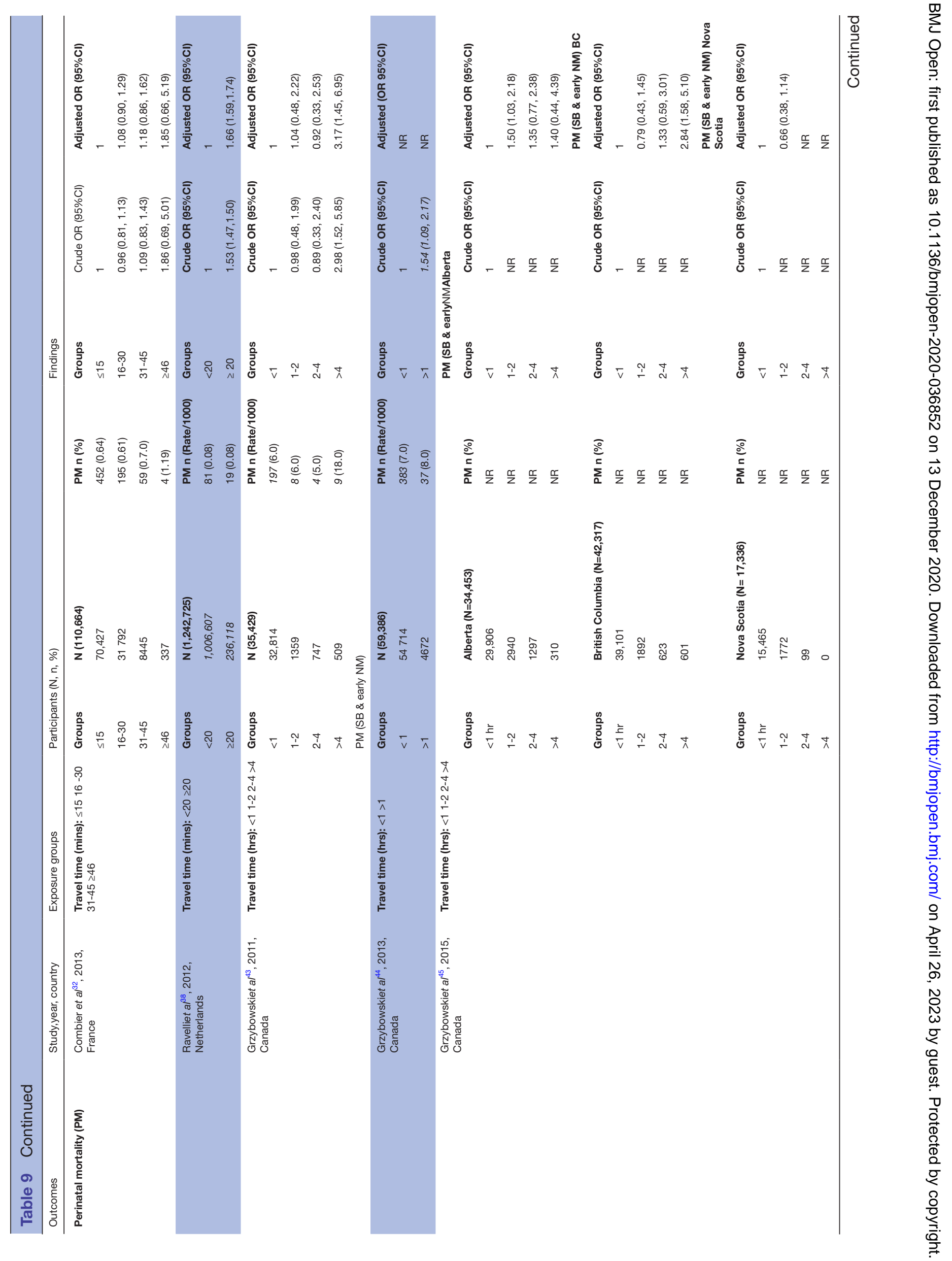




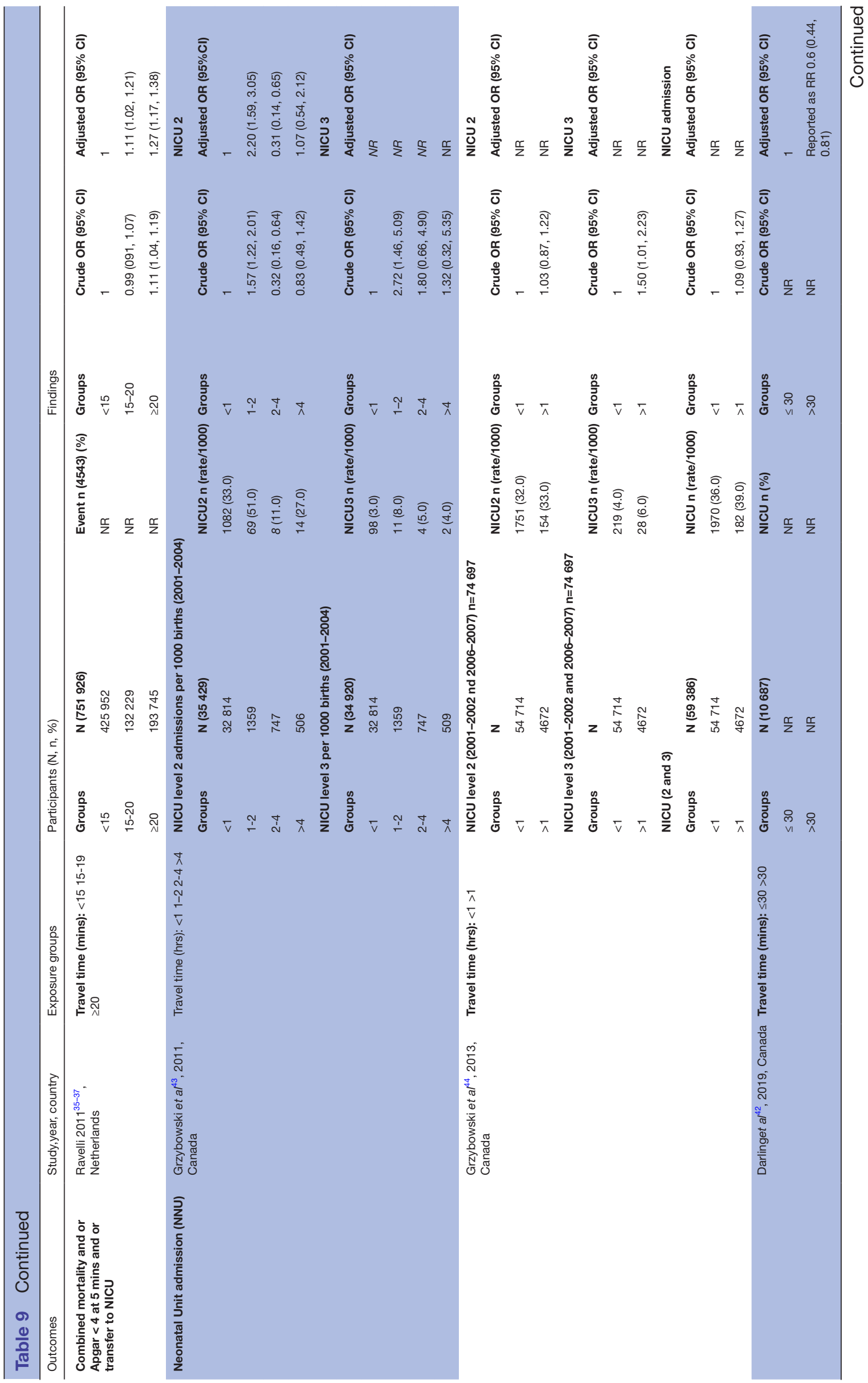




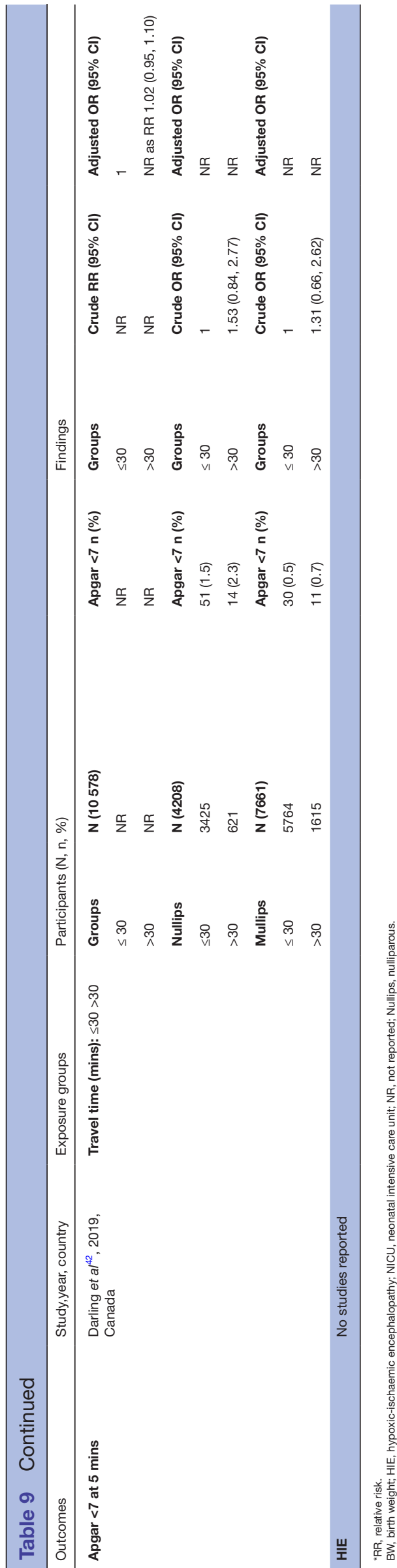

living more than 2 hours away from an OU compared with women living less than 1 hour from an OU based on an unadjusted analysis (cOR 3.57, 95\% CI 0.81 to 15.76 ). In Darling $e t a l,{ }^{42}$ the PM rates were not statistically significantly different for women with a planned home birth and more than $30 \mathrm{~min}$ drive from hospital (adjRR 2.2, $95 \%$ CI 0.67 to 7.43 ).

Infant mortality (IM)

One Canadian study ${ }^{43}$ reported no significant difference in IM rates for women living less than 1-hour travel time to OU compared with more than 1-hour travel time to OU (cOR $1.51,95 \%$ CI 0.86 to 2.63 ).

\section{Born before arrival (BBA)}

Six studies reported this outcome, four cohort studies $^{32} 414344$ and two case-control studies. ${ }^{33} 34$ Five of the six studies found some association between travel time and BBA, four based on adjusted analyses.

There were three studies conducted in France. ${ }^{32-34}$ Combier $e t a l,{ }^{32}$ reported that a travel time greater than 15 min was significantly associated with an increased risk of BBA (16-30 min vs < 16 min, adjOR $1.73,95 \%$ CI 1.23 to 2.46 ); (31-45 $\mathrm{min}$ vs $<16 \mathrm{~min}$, adjOR $1.64,95 \%$ CI 1.06 to 2.54$).{ }^{32}$ In a case-control study, ${ }^{34}$ the BBA rate increased sixfold when the travel time increased to more than 45 min from home to the OU compared with women who travelled less than $15 \mathrm{~min}(>45 \mathrm{~min}$ vs $>15$ min, adjOR $6.1895 \%$ CI 1.33 to 28.65). However, in the other case- control study the risk of BBA was not significantly increased in women who travelled for greater than 20 min. $^{33}$

In a study from Norway, ${ }^{40}$ the risk of BBA increased significantly with longer travel time to the nearest $\mathrm{OU}$ from home. Women who travelled more than 2 hours had an eight fold increased risk of BBA compared with women who lived within 1 hour of the nearest OU $(>2$ hours vs $<1$ hour, cOR $8.21,95 \%$ CI 7.19 to 9.37$).{ }^{41}$

The studies from Canada ${ }^{43}$ found a significant increase in BBA in women living in communities greater than 1-hour travel time from an OU compared with those living less than 1 hour away. In Grzybowski et al, ${ }^{43}$ women who lived 1-2 hours from an OU had the highest risk of BBA compared with less than 1 hour (adjOR 6.41, 95\% CI 3.69 to 11.28) and women who lived greater than 4 hours away also had an increased risk compared with those living less than 1 hour away (adjOR 3.63, 95\% CI 1.40 to 9.40 ); however, there was no difference between those who lived 2-4 hours from an OU and those living less than 1 hour away (adjOR $0.92,95 \%$ CI 0.22 to 3.88 ). Gryzbowski $e t a l,{ }^{44}$ found a five-=fold increase in BBA in women who lived more than an hour away from an OU in comparison to women who lived less than an hour away (cOR 5.06, 95\% CI 3.82 to 6.70 ).

Neonatal unit (NNU) admission

Three studies from Canada reported on NNU admission. ${ }^{42-44}$ The two studies from British Columbia ${ }^{43} 44$ 
reported NNU depending on whether the admission was for level 2 care (high dependency) or level 3 care (intensive care). Findings from one of these studies ${ }^{43}$ showed NNU level 2 admission increased significantly in babies born to women living more than 1 hour away from an OU compared with less than 1 hour (adjOR 2.20, 95\% CI 1.59 to 3.05). For those living 2-4 hours away, level 2 admissions were significantly lower compared with those living less than 1 hour away (adjOR 0.31, 95\% CI 0.14 to 0.65$)$. For those living more than 4 hours away, there appeared to be no increase in NNU level 2 admission. For level 3 NNU admission, a significantly increased risk was found for the 1-2 hours category (1-2 hours vs $<1$ hour, cOR 2.72, 95\% CI 1.46 to 5.09). For the other two categories, 2-4 and $>4$ hours, neither crude nor adjusted analyses showed any significant difference. The number of women in each group decreased with increasing time from an OU. In Grzybowski $e t a l,{ }^{44}$ there was no increased risk of admission to NNU level 2 in babies born to women living more than 1 hour from an OU compared with less than 1 hour, however, admission to NNU level 3 was significantly higher (cOR $1.50,95 \%$ CI 1.01 to 2.23 ). The third Canadian study from Ontario ${ }^{42}$ showed a lower relative risk of NNU admission for planned home births with a travel time greater than 30 min when compared with less than 30 min (adjRR 0.6, 95\% CI 0.44 to 0.81 ).

\section{Apgar score}

Two studies reported on Apgar Score; one from Canada and one from the Netherlands. ${ }^{372}$ In the Canadian study, ${ }^{42}$ no significant difference was found for Apgar score $<7$ at 5 min between women who planned home birth and lived more or less than 30 min away from an OU, either for nulliparous or multiparous subgroups (adjRR $1.02,95 \%$ CI 0.95 to 1.10$)$.

The study from the Netherlands ${ }^{37}$ used a composite outcome of mortality and/or Apgar $<4$ at 5 min and/ or transfer to NNU, and showed a small but significant increase in this outcome in women whose travel time to an OU exceeded $15 \mathrm{~min}$ (15-20 min vs $<15 \mathrm{~min}$, adjOR $1.11,95 \%$ CI 1.02 to 1.21 and $\geq 20 \mathrm{~min}$ vs $<15 \mathrm{~min}$, adjOR $1.27,95 \%$ CI 1.17 to 1.38$)$.

Hypoxic-ischaemic encephalopathy (HIE)

No studies reported this outcome.

\section{DISCUSSION}

This review describes studies which have explored the associations between OU closure, distance or travel time to an OU, and maternal and neonatal outcomes. The included studies were conducted in the UK, France, the Netherlands, Norway, Canada and Japan. Many studies were from parts of the world where service configuration varied and the study populations were sometimes dispersed over a large geographical area. The included studies differed in their design, geographical boundaries, outcomes measures used and included a wide range of travel time/distance thresholds used. In addition, although many studies reported that potential confounders were adjusted for in their analyses, many of the outcomes of interest for this review were crude measures of effect without adjustment. Therefore, comparing these studies with each other was a challenge.

All of these studies were brought together to explore whether women who had to travel longer and further to their planned OU were at increased risk of adverse outcomes. There was one reasonably consistent finding which was that there appeared to be an increased risk of BBA the longer it took to reach the OU. This may have been associated with an increased risk for the baby with a suggestion of an increased risk of perinatal or NM in some studies, however, this effect was not consistent across all the studies. There was also an increase in CS rates following closure of an OU and with shorter travel distance and time, however, it is unclear if the difference was related to the exposure or unmeasured differences in CS rates.

\section{Strengths and limitations of the review}

This work is the first to synthesise systematically the current evidence relevant to OU closure and the impact of travel time and travel distance on maternal and neonatal outcomes. Rigorous systematic review methodology was applied, including a sensitive search strategy to identify all the relevant literature, and thorough assessment of potential risks of bias. All screening, data extraction and risk of bias assessment were performed independently by at least two reviewers.

The process of selecting studies for inclusion was challenging due to a lack of reporting of some details, for example, it was not always clear which level of maternity services the study referred to, in others, findings related to the impact of travel time and distance were not always presented despite this being described as a study objective.

\section{INTERPRETATION OF FINDINGS}

It is difficult to conclude from this review whether reconfiguration of maternity services, with closure of OUs, resulting in increased travel distances and times for women is unequivocally associated with worse outcomes for the mother or the baby. Assessing the impact of OU closure and prolonged travel time and distance is not straightforward; to isolate the impact of the closure and travel time and distance on maternal and neonatal outcomes we need to fully understand the models of maternity care, transport services, landscape characteristics, women's satisfaction with care and places of birth available to women in that specific geographical area. Understanding how services are delivered to women is vital when assessing the impact of travel distance and time as services may be adapted to meet the challenges for women living in remote areas, for example by transferring women antenatally a few weeks before birth. Some studies found an increase in CS rates with shorter 
distance/travel time. Attributing this solely to closure or reconfiguration of services is problematic as simple analytical comparisons of rates before and after changes do not account for underlying time trends. Future studies might want to consider an interrupted time series design as a more appropriate method.

There remains an urgent need to evaluate the impact of changing maternity service provision. The imperative to close and consolidate OUs into larger units is based on a belief that this will improve safety for both mother and baby. If increasing travel times and distances increases risks to mothers and babies, then the postulated benefits of larger OUs could be offset by the harms of the reconfiguration.

Waiting for closure of OUs to prospectively evaluate the impact on the surrounding maternity population will always be challenging. However, exploring the existing impact of distance and travel time from home to an OU may be a reasonable approach to explore what the impact of reconfiguration may be for a proportion of the women in the area served by the OU which would have these parameters increased by closure of one of more local OU (s). Such a study would need to be large to explore the impact of travel time and distance on substantive harms such as mortality for the baby, so will almost certainly need to use routinely collected data to obtain large numbers. Such studies will also need to include vigorous evaluation of confounders, such as maternal characteristics, socioeconomic status and maternal medical history, which are known to influence birth outcomes; controlling for these factors is vital to determine the OU closure impacts. These studies should also collect data at multiple time points after the closure and apply statistical analysis which considers time-varying relationships and the outcomes.

Measurement of travel time and distance from the woman's place of residence to an OU would also need more sophisticated approaches than previously used in many studies; for example the use of web-based route planners and adjustment for travel conditions rather than using straight line distances or relying on self-reports.

Many study designs assume that travel time and distance have a constant effect on outcomes. If local OUs are far away, it is possible that women will modify their behaviour in relation to when they set off for their OU in labour, if they know they have an hour's journey compared with a 20 min journey. The extent to which this will mitigate the effects of longer travel times would not be seen in a study looking at existing travel times and distances.

\section{CONCLUSION}

Given the substantial variation across studies we were unable to draw firm conclusions regarding the association between OU closure, travel distance or time to obstetric services and maternal and neonatal outcomes. There appears to be a consistent association with BBA with increasing distance and travel time to an $\mathrm{OU}$ and a suggestion of increasing risk to the baby. However, few studies have rigorously controlled for potential confounders.

\section{Twitter Charles Opondo @charlesopondo}

Acknowledgements Our thanks to Pamela White for contacting NHS Trusts and obtaining papers, Nia Roberts for development of the search strategy and Mark Willett and Fiona Mackie for providingun published data.

\section{Collaborators NA.}

Contributors PB, FA, RSM and JD conceived the research. All authors developed the protocol and RSM developed the search strategy. RSM, CT, AP, FA and JH screened the search results and full papers. RSM, CT, JH, FA and CO assessed the quality of included papers, extracted the data and synthesised the results. RSM and FA drafted the manuscript and all authors agreed the final manuscript.

Funding This research is funded by the National Institute for Health Research (NIHR) Policy Research Programme, conducted through the Policy Research Unit in Maternal Health and Care, 108/0001.

Disclaimer The views expressed are those of the author(s) and not necessarily those of the NIHR or the Department of Health and Social Care.

Competing interests None declared.

Patient consent for publication Not required.

Provenance and peer review Not commissioned; externally peer reviewed.

Data availability statement All data relevant to the study are included in the article or uploaded as online supplemental information. All the data included in this systematic review are in the public domain.

Supplemental material This content has been supplied by the author(s). It has not been vetted by BMJ Publishing Group Limited (BMJ) and may not have been peer-reviewed. Any opinions or recommendations discussed are solely those of the author(s) and are not endorsed by BMJ. BMJ disclaims all liability and responsibility arising from any reliance placed on the content. Where the content includes any translated material, BMJ does not warrant the accuracy and reliability of the translations (including but not limited to local regulations, clinical guidelines, terminology, drug names and drug dosages), and is not responsible for any error and/or omissions arising from translation and adaptation or otherwise.

Open access This is an open access article distributed in accordance with the Creative Commons Attribution 4.0 Unported (CC BY 4.0) license, which permits others to copy, redistribute, remix, transform and build upon this work for any purpose, provided the original work is properly cited, a link to the licence is given, and indication of whether changes were made. See: https://creativecommons.org/ licenses/by/4.0/.

\section{ORCID iDs}

Reem Saleem Malouf http://orcid.org/0000-0002-0673-5126

Charles Opondo http://orcid.org/0000-0001-8155-4117

\section{REFERENCES}

1 Jordan H, Roderick P, Martin D, et al. Distance, rurality and the need for care: access to health services in South West England. Int J Health Geogr 2004;3:21.

2 Kelly C, Hulme C, Farragher T, et al. Are differences in travel time or distance to healthcare for adults in global North countries associated with an impact on health outcomes? A systematic review. BMJ open 2016;6:e013059.

3 Rashidian A, Omidvari AH, Vali Y, et al. The effectiveness of regionalization of perinatal care services-a systematic review. Public Health 2014;128:872-85.

4 Price S, Little S. Research evidence review -impact of distance/travel time to maternity services on birth outcomes.Public Health Wales NHS Trust; 2015. http://www.publichealthwales.org/maternityreview [Accessed January 2019].

5 Blondel B, Drewniak N, Pilkington H, Pilkington Nicolas; , Hugo; Zeitlin J, et al. Out-Of-Hospital births and the supply of maternity units in France. Health Place 2011;17:1170-3.

6 Lasswell SM, Barfield WD, Rochat RW, et al. Perinatal regionalization for very low-birth-weight and very preterm infants: a meta-analysis. JAMA 2010;304:992-1000. 
7 Engjom HM, Morken N-H, Norheim OF, et al. Availability and access in modern obstetric care: a retrospective population-based study. BJOG 2014;121:290-9.

8 Stroup DF, Berlin JA, Morton SC, et al. Meta-Analysis of observational studies in EpidemiologyA proposal for reporting. JAMA 2000;283:2008-12.

9 Merenstein GB, Glicken AD. Best evidence-based practices: a historic perspective. Neonatal Netw 2002;21:31-5.

10 World Health Organization. Universal health coverage (UHC), 2019. Available: https://wwwwhoint/news-room/fact-sheets/detail/ universal-health-coverage-(uhc)

11 Rowe R, McCourt C, MacFarlane A, The Birthplace in England Collaborative Group. Birthplace terms and definitions: consensus process Birthplace in England research programme.(Final Report 2. 08/1604/140). Southampton: HMSO, 2011. http:// openaccesscityacuk/3651/1/Birthplace\%20definitions\%20rpt\%20 SDO_FR2_08-1604-140_V02pdf

12 Thomas J, Graziosi S. EPPI-Reviewer: advanced software for systematic reviews, maps and evidence synthesis 2020;4.

13 Cochrane Effective Practice and Organisation of Care (EPOC). Suggested risk of bias cri teria for EPOC reviews. EPOC Resources for review authors, 2017. Available: ]http://ep occochraneorg/res ources /epoc-res ources-revi ew-authors

14 Stang A. Critical evaluation of the Newcastle-Ottawa scale for the assessment of the quality of nonrandomized studies in metaanalyses. Eur J Epidemiol 2010;25:603-5.

15 Fleming AM, Martindale EE, Schram C. Reducing caesarean section rates through choice and collaboration. Archives of disease in childhood: fetal and neonatal edition conference: 16th annual conference of the British maternal and fetal medicine Society Dublin Ireland conference publication. Archives of Disease in Childhood Fetal and Neonatal Edition 2 2013;98:A55-6.

16 Mackie FLA, Moise F, Amu O. Maternal and neonatal outcomes after the amalgamation of two maternity units and consequent increased consultant labour ward presence: a retrospective population-based study. Archives of Disease in Childhood: Fetal and Neonatal Edition 2014;99:A21-2.

17 Grytten J, Monkerud L, Skau I, et al. Regionalization and local hospital closure in Norwegian maternity care--the effect on neonatal and infant mortality. Health Serv Res 2014;49:1184-204.

18 Hemminki E, Heino A, Gissler M. Should births be centralised in higher level hospitals? experiences from regionalised health care in Finland. BJOG 2011;118:1186-95.

19 Allen VM, Jilwah N, Joseph KS, et al. The influence of hospital closures in nova Scotia on perinatal outcomes. Journal of Obstetrics and Gynaecology Canada 2004;26:1077-85.

20 Hutcheon JA, Riddell CA, Strumpf EC, et al. Safety of labour and delivery following closures of obstetric services in small community hospitals. CMAJ 2017;189:E431-6.

21 Le Coutour X, Infante-Rivard C, Danzon A. [Regionalization of health care and obstetric practice]. Rev Epidemiol Sante Publique 1990;38:211-20.

22 Allen VM, Jilwah N, Joseph KS, et al. The influence of hospital closures in nova Scotia on perinatal outcomes. J Obstet Gynaecol Can 2004;26:1077-85

23 Bhoopalam PS, Watkinson M. Babies born before arrival at hospital. Br J Obstet Gynaecol 1991;98:57-64.

24 Pasquier J-C, Morelle M, Bagouet S, Moret S.; et al. Effects of residential distance to hospitals with neonatal surgery care on prenatal management and outcome of pregnancies with severe fetal malformations. Ultrasound Obstet Gynecol 2007;29:271-5.

25 Pilkington $\mathrm{H}$, Blondel B, Drewniak N, Zeitlin J, et al. Where does distance matter? distance to the closest maternity unit and risk of foetal and neonatal mortality in France. Eur J Public Health 2014;24:905-10.

26 Fougner B, Nakling J. [Deliveries during transportation and shortly after admission to hospital]. Tidsskr Nor Laegeforen 2000;120:1288-90.

27 Ovaskainen K, Ojala R, Gissler M, et al. Out-Of-Hospital deliveries have risen involving greater neonatal morbidity: risk factors in out-of- hospital deliveries in one university hospital region in Finland. Acta Paediatr 2015;104:1248-52.

28 Lisonkova S, Sheps SB, Janssen PA, et al. Birth outcomes among older mothers in rural versus urban areas: a residence-based approach. J Rural Health 2011;27:211-9.

29 Dummer TJB, Parker L. Hospital accessibility and infant death risk. Arch Dis Child 2004;89:232-4.

30 Paranjothy SW, Gong K, et al. Perinatal outcomes and travel time to maternity services: analysis of birth outcome data in Wales from 1995 to 2009. Archives of disease in childhood: fetal and neonatal edition conference: 16th annual conference of the British maternal and fetal medicine Society Dublin Ireland conference publication: 2013; 98, 2013.

31 Paranjothy S, Watkins WJ, Rolfe K, et al. Perinatal outcomes and travel time from home to hospital: Welsh data from 1995 to 2009. Acta Paediatr 2014;103:e522-7.

32 Combier E, Charreire H, Le Vaillant M, et al. Perinatal health inequalities and accessibility of maternity services in a rural French region: closing maternity units in Burgundy. Health Place 2013;24:225-33.

33 Nguyen M-L, Lefèvre P, Dreyfus M. [Maternal and neonatal outcomes of unplanned deliveries]. J Gynecol Obstet Biol Reprod 2016;45:86-91.

34 Renesme L, Garlantézec R, Anouilh F, et al. Accidental outof-hospital deliveries: a case-control study. Acta Paediatr 2013;102:e174-7.

35 Ravelli A, K. J.; De, Groot; M, et al. Travel time from home to hospital and intrapartum and neonatal mortality in term pregnant women. Journal of Maternal-Fetal and Neonatal Medicine 2010;23:234.

36 Ravelli A, K. J.; De, Groot; M, et al. Travel time from home to hospital and adverse perinatal outcomes in women at term in the Netherlands. Obstetrical and Gynecological Survey 2011;66:396-8.

37 Ravelli A, K. J.; de, Groot; M, et al. Travel time from home to hospital and adverse perinatal outcomes in women at term in the Netherlands. BJOG: An International Journal of Obstetrics \& Gynaecology 2011;118:457-65.

38 Ravelli AEM, Erwich J, Brouwers HAA, et al. H.O.PC., Neoned* \& B.W.J. Mol. Perinatale sterfteverschillen in de negen perinatale zorgregio's Nederlands tijdschrift voor. Obstetrie \& Gynaecologie 2012;125:270-7.

39 Stolp I, Smit M, Luxemburg S, et al. Ambulance transfer in case of postpartum hemorrhage after birth in primary midwifery care in the Netherlands: a prospective cohort study. Birth 2015;42:227-34.

40 Engjom HM NH, Hoydal E, Norheim O. Obstetric health system structure and perinatal outcomes in Norway. Int $J$ Gynaecol Obstet 2015;131:E487-8.

41 Engjom HM, Morken N-H, Høydahl E, et al. Increased risk of Peripartum perinatal mortality in unplanned births outside an institution: a retrospective population-based study. Am J Obstet Gynecol 2017;217:210.e1-2

42 Darling EK, Lawford KMO, Wilson K, et al. Distance from home birth to emergency obstetric services and neonatal outcomes: a cohort study. J Midwifery Womens Health 2019;64:170-8.

43 Grzybowski S, Stoll K, Kornelsen J. Distance matters: a population based study examining access to maternity services for rural women. BMC Health Serv Res 2011;11:147.

44 Grzybowski S, Stoll K, Kornelsen J. The outcomes of perinatal surgical services in rural British Columbia: a population-based study. Can J Rural Med 2013;18:123-9.

45 Grzybowski S, Fahey J, Lai B, Lai John; Zhang Barbara; , et al. The safety of Canadian rural maternity services: a multi-jurisdictional cohort analysis. BMC Health Serv Res 2015;15:410.

46 Stoll K, Kornelsen J. Midwifery care in rural and remote British Columbia: a retrospective cohort study of perinatal outcomes of rural parturient women with a midwife involved in their care, 2003 to 2008. J Midwifery Womens Health 2014;59:60-6.

47 Aoshima K, Kawaguchi $\mathrm{H}$, Kawahara K. Neonatal mortality rate reduction by improving geographic accessibility to perinatal care centers in Japan. J Med Dent Sci 2011;58:29-40. 